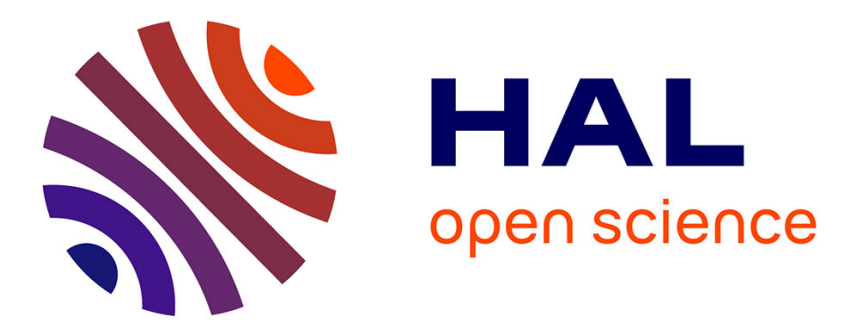

\title{
Les structures linéaires fossoyées issues de l'archéologie préventive dans l'Est lyonnais : essai de synthèse Catherine Coquidé
}

\section{To cite this version:}

Catherine Coquidé. Les structures linéaires fossoyées issues de l'archéologie préventive dans l'Est lyonnais: essai de synthèse. Revue archéologique de Narbonnaise, 2003, 36 (1), pp.7-24. 10.3406/ran.2003.1115 . halshs-01966986

\section{HAL Id: halshs-01966986 \\ https://shs.hal.science/halshs-01966986}

Submitted on 30 Dec 2018

HAL is a multi-disciplinary open access archive for the deposit and dissemination of scientific research documents, whether they are published or not. The documents may come from teaching and research institutions in France or abroad, or from public or private research centers.
L'archive ouverte pluridisciplinaire HAL, est destinée au dépôt et à la diffusion de documents scientifiques de niveau recherche, publiés ou non, émanant des établissements d'enseignement et de recherche français ou étrangers, des laboratoires publics ou privés. 


\title{
Les structures linéaires fossoyées issues de l'archéologie préventive dans l'Est lyonnais : essai de synthèse
}

In: Revue archéologique de Narbonnaise, Tome 36, 2003. pp. 7-24.

\begin{abstract}
The preventive archaeological excavations in the East of Lyons have increased the discoveries of linear lines. This synthetis gives them a place in two studied areas covering several hectares. The second part increased with other sites explains the chronological heads available, brings out recurrent positionings, emphasizes the topography and the human activities effects. Finally, this paper recalls the hypothesises about centuratio expressed in this district. A comparison with Napoleon's cadastre brings to light the origin of many parcel anomalies owing to the selection and the superimposition of divergent layouts. A new explanation is given to the lines composed of pebble trenches (agrarian divisions ?).

\section{Résumé}

Les décapages de l'archéologie préventive dans l'Est lyonnais ont multiplié les mises au jour de tracés linéaires. Cette synthèse leur redonne une place dans deux zones d'études couvrant plusieurs dizaines d'hectares. Augmentée d'autres sites, la seconde partie expose les pistes chronologiques disponibles, met en valeur des orientations récurrentes, souligne l'influence de la topographie et des occupations humaines. Enfin, l'article évoque les hypothèses de centuriation émises dans ce secteur. Une confrontation avec le cadastre napoléonien illustre l'origine de nombreuses anomalies parcellaires due à la sélection et à la surimposition de tracés divergents. Les lignes de fosses à galets, éventuelles divisions agraires, y trouvent également un éclairage nouveau.
\end{abstract}

Citer ce document / Cite this document :

Coquidé Catherine. Les structures linéaires fossoyées issues de l'archéologie préventive dans l'Est lyonnais : essai de synthèse. In: Revue archéologique de Narbonnaise, Tome 36, 2003. pp. 7-24.

doi : 10.3406/ran.2003.1115

http://www.persee.fr/web/revues/home/prescript/article/ran_0557-7705_2003_num_36_1_1115 


\title{
Les structures linéaires fossoyées issues de l'archéologie préventive dans l'Est lyonnais : essai de synthèse
}

\author{
Catherine COQUIDÉ *
}

\begin{abstract}
Résumé : Les décapages de l'archéologie préventive dans l'Est lyonnais ont multiplié les mises au jour de tracés linéaires. Cette synthèse leur redonne une place dans deux zones d'études couvrant plusieurs dizaines d'hectares. Augmentée d'autres sites, la seconde partie expose les pistes chronologiques disponibles, met en valeur des orientations récurrentes, souligne l'influence de la topographie et des occupations humaines. Enfin, l'article évoque les hypothèses de centuriation émises dans ce secteur. Une confrontation avec le cadastre napoléonien illustre l'origine de nombreuses anomalies parcellaires due à la sélection et à la surimposition de tracés divergents. Les lignes de fosses à galets, éventuelles divisions agraires, y trouvent également un éclairage nouveau.

Mots-clés : Est lyonnais, archéologie préventive, fossés, fosses à galets, limites parcellaires, réseaux, orientations, cadastres, centuriation.

Abstract : The preventive archaeological excavations in the East of Lyons have increased the discoveries of linear lines. This synthetis gives them a place in two studied areas covering several hectares. The second part increased with other sites explains the chronological heads available, brings out recurrent positionings, emphasizes the topography and the human activities effects. Finally, this paper recalls the hypothesises about centuratio xpressed in this district. A comparison with Napoleon's cadastre brings to light the origin of many parcel anomalies owing to the selection and the iuperimposition of divergent layouts. A new explanation is given to the lines composed of pebble trenches (agrarian divisions ?).

Keywords : East of Lyons, preventive archaeology, ditches, pebble trenches, parcel boundaries, network, position, cadastre, centuratio.
\end{abstract}

\section{Introduction}

Durant la dernière décennie, le déveLoppement de la couronne est lyonnaise a généré un grand nombre d'études d'impact archéologique et de fouilles. Celles-ci ont pris place sur une même entité géologique, la plaine de l'Est lyonnais, limitée par la côtière des Dombes au nord, à l'est par le plateau calcaire de l'Isle Crémieu, au sud par les Balmes viennoises et à l'ouest par les massifs primaires des Monts du Lyonnais. Cette plaine d'environ $25 \mathrm{~km}$ sur 30 présente un relief contrasté. C'est une étendue monotone, modelée par les avancées et les reculées glaciaires, incisée par un fleuve et quelques rivières, mais ponctuée de buttes et de plateaux morainiques aux pentes parfois marquées. La partie orientale de cette surface qui est moins concernée par les aménagements reste beaucoup moins renseignée, ce déséquilibre est/ouest reflétant l'état de la recherche (fig. 1 et 2).

De nombreux fossés ont été mis au jour lors de ces décapages. L'interprétation des plans de masse ne livre souvent que des informations incomplètes sur la donnée de base que constitue le fossé : un tracé, une orientation et une éventuelle surimposition avec d'autres segments

linéaires ou parfois une imbrication dans l'occupation humaine. Souvent, la trace au sol ne révèle pas sa ou ses fonctions et il faut admettre qu'il est souvent impossible d'identifier et a fortiori de hiérarchiser l'importance du fossé au sein d'un site, de distinguer un réseau d'un tracé isolé, de dissocier les éléments fondateurs des développements ultérieurs ou même d'évaluer leur portée spatiale dans la gestion des sols. A l'échelle du chantier, l'interprétation se limite donc, au pire, à une simple mention de 

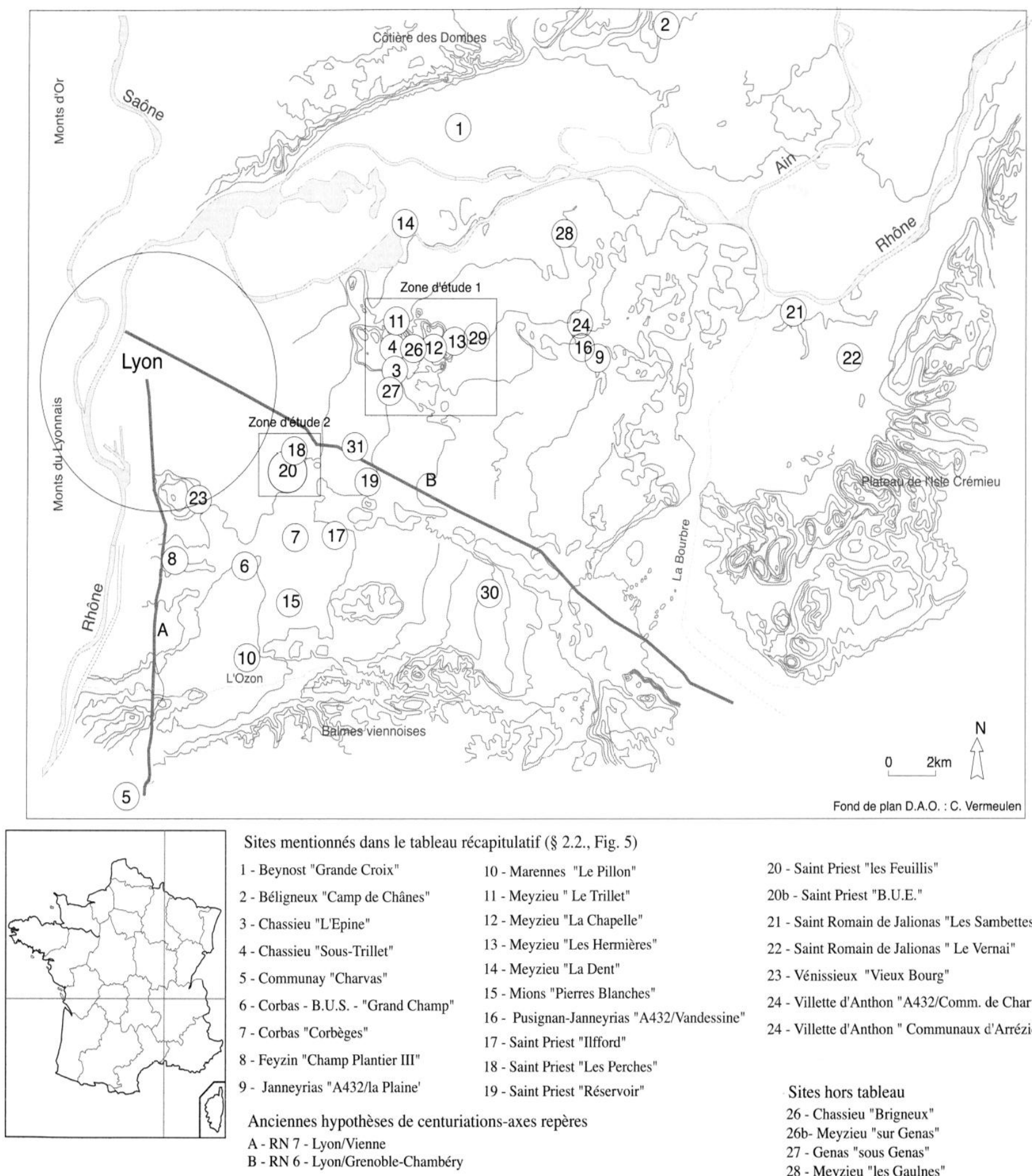

20 - Saint Priest "les Feuillis"

20 b - Saint Priest "B.U.E."

21 - Saint Romain de Jalionas "Les Sambettes"

22 - Saint Romain de Jalionas " Le Vernai"

23 - Vénissieux "Vieux Bourg"

24 - Villette d'Anthon "A432/Comm. de Charvas"

24 - Villette d'Anthon "Communaux d'Arrézieux"

Sites hors tableau

26 - Chassieu "Brigneux"

26b- Meyzieu "sur Genas"

27 - Genas "sous Genas"

28 - Meyzieu "les Gaulnes"

29 - Meyzieu "les Tâches"

30 - Saint Laurent de Mure "la Grande Cr,"

31 - Saint Priest "Champ Dolin"

\section{Fig. 2. Est lyonnais : localisation des sites mentionnés}

vestige dénué de contexte. Au mieux, quand l'environnement est mieux documenté (imbrication avec l'occupation, apparente cohérence de l'implantation, matériel datant), l'information prend de l'ampleur et le fossé peut trouver sa place dans l'une ou l'autre des phases de l'évolution du site. Sur le terrain, se regroupent donc sous le terme générique de "fossés" tous les tracés, quels qu'en soient les profils, d'ailleurs variables à l'intérieur d'un même vestige, l'orientation générale restant la donnée commune la plus fiable.

Toutes ces questions sans réponse ont parfois conduit à reléguer ces structures linéaires fossoyées au second plan 
et, bien qu'on en pressente l'intérêt en terme d'emprise de l'homme sur un terroir, à les exclure des interprétations finales.

Dans le même temps, des travaux issus d'une autre source documentaire ont connu un essor considérable : la lecture de l'évolution des formes du paysage révélées par la carto- et la photo-interprétation. Cette enquête à plus grande échelle retient pour hypothèse de base la conservation au moins partielle de certaines caractéristiques des réseaux anciens dans les limites agraires actuelles. A l'origine, c'est la présence/absence de centuriations, forme antique et normalisée de cadastration des terres, qui a focalisé l'attention dans les régions les plus méridionales. Bien que la recherche n'ait cessé d'évoluer, bon nombre de réflexes sont restés piégés à l'intérieur de ce schéma et la référence à des hypothèses anciennes telles que les "centuriations de l'Est lyonnais" a oblitéré, voire paralysé, tout autre type d'observation.

Cet essai est une première synthèse des données de fouilles pour elles-mêmes, à partir de la seule lecture des plans de masse '. Dans cette optique, l'étude dépasse le cadre du site et se tourne vers l'ensemble de la plaine de l'Est lyonnais. Elle rassemble les données disponibles, les croise et en recherche d'éventuels points communs, ou le cas échéant, en note les profondes disparités. A ce titre, la confrontation avec les documents graphiques (cadastre napoléonien ou missions aériennes) est une piste parmi d'autre, un éclairage différent à verser au bénéfice de l'interprétation des sites où la recherche d'éventuelles centuriations ne doit plus être le seul objectif.

L'article présente donc deux zones d'étude ayant bénéficié de dizaines d'hectares de sondages et de fouilles livrant ainsi de nombreux tracés linéaires fossoyés (Rocade Est/Contournement sud de Meyzieu (zone d'étude 1) et Boulevard Urbain Est/Zac des Feuillis (zone d'étude 2). A ces structures linéaires fossoyées, l'étude adjoint un type de vestige particulier : des alignements de fosses à galets dont les agencements décelés sur de grandes distances et les profils permettent de réfuter l'hypothèse des pierriers et de s'orienter vers une technique originale de limitation des sols. Puis, élargissant le cadre à d'autres opérations d'emprise au sol plus modeste, l'étude constatera quelques traits communs à ce large éventail d'éléments disparates. Enfin, après une comparaison des résultats avec la recherche sur les systèmes par- cellaires de l'Est lyonnais issue de la carto-et photo-interprétation, les plans de masse ont été confrontés aux cadastres napoléoniens à la recherche d'éventuels points de rencontre.

\section{Décapages arcchéologiques et structures linéaires : études de cas}

Deux secteurs de l'agglomération lyonnaise ont bénéficié d'un décapage sur plusieurs dizaines d'hectares au contact des buttes et de la plaine mettant au jour de nombreux fossés. Il s'agit d'une part, des études archéologiques de la rocade est et du contournement sud de Meyzieu sur les communes de Chassieu, Genas, Meyzieu et Décines et d'autre part de la ZAC des Feuillis et du Boulevard Urbain Est (BUE) à Saint-Priest.

Après une rapide présentation morphologique des lieux, chaque groupe d'orientations est présenté associé aux informations archéologiques disponibles : situation par rapport aux autres vestiges ou éventuelle indication chronologique. Concernant ce dernier point, les données (matériel en contexte ou relation stratigraphique) sont restées rares et doivent être considérées avec prudence. Il convient également de rappeler que les orientations suivantes ne correspondent en aucun cas à un plan d'implantation parcellaire reconnu et n'ont été numérotées que pour servir de repères dans le texte.

\subsection{La butte morainique de Chassieu, Genas, Meyzieu et Décines : deux visions transversales, d'un même relief (zone d'étude 1)}

La rocade est et le contournement sud de Meyzieu quadrillent une même entité morphologique, l'un en estouest et l'autre en nord-sud représentent près de 8 kilomètres linéaires qui ont été intégralement sondés avant d'être décapés sur près de 10 hectares 2 (fig. 3).

La butte morainique concernée par cette première étude est située sur le quart nord-ouest de la plaine. Son orientation nord-ouest/sud-est témoigne des anciennes divagations de masses d'eau liées aux différents mouvements glaciaires. D'une longueur maximale de $7 \mathrm{~km}$ et d'une largeur de $4 \mathrm{~km}$, elle culmine à $250 \mathrm{~m}$ et surplombe une plaine s'étageant de 210 à $190 \mathrm{~m}$ d'altitude. Son soussol, principalement morainique, est par endroits recouvert

1 Les rapports de sondages ou les documents finaux de synthèse sont listés en fin d'article. Les plans de masse ont permis de mesurer les axes de limites linéaires variées et des perpendiculaires ont été tracées afin de rendre les comparaisons effectives par rapport au nord. L'enregistrement électronique puis numérique des données étant encore récent, nous sommes conscients des limites de ce support. En effet, bon nombre de plans ont pu subir des distorsions dues au relevé, au papier ou à la reprographie. Le nord Lambert a été notre principal repère bien que certains documents ne soient pas clairs à ce propos.

2 Archéologues responsables d’opération (AFAN) : C. Coquidé "L’Epine " "la Chapelle " " les Taches " " Brigneux "; P. Jacquet " les Hermières " "Sur Genas" : D. Frascone "le Trillet" : J.-L. Joly "Sous-Trillet " : J. Vital " Genas sous Genas". 


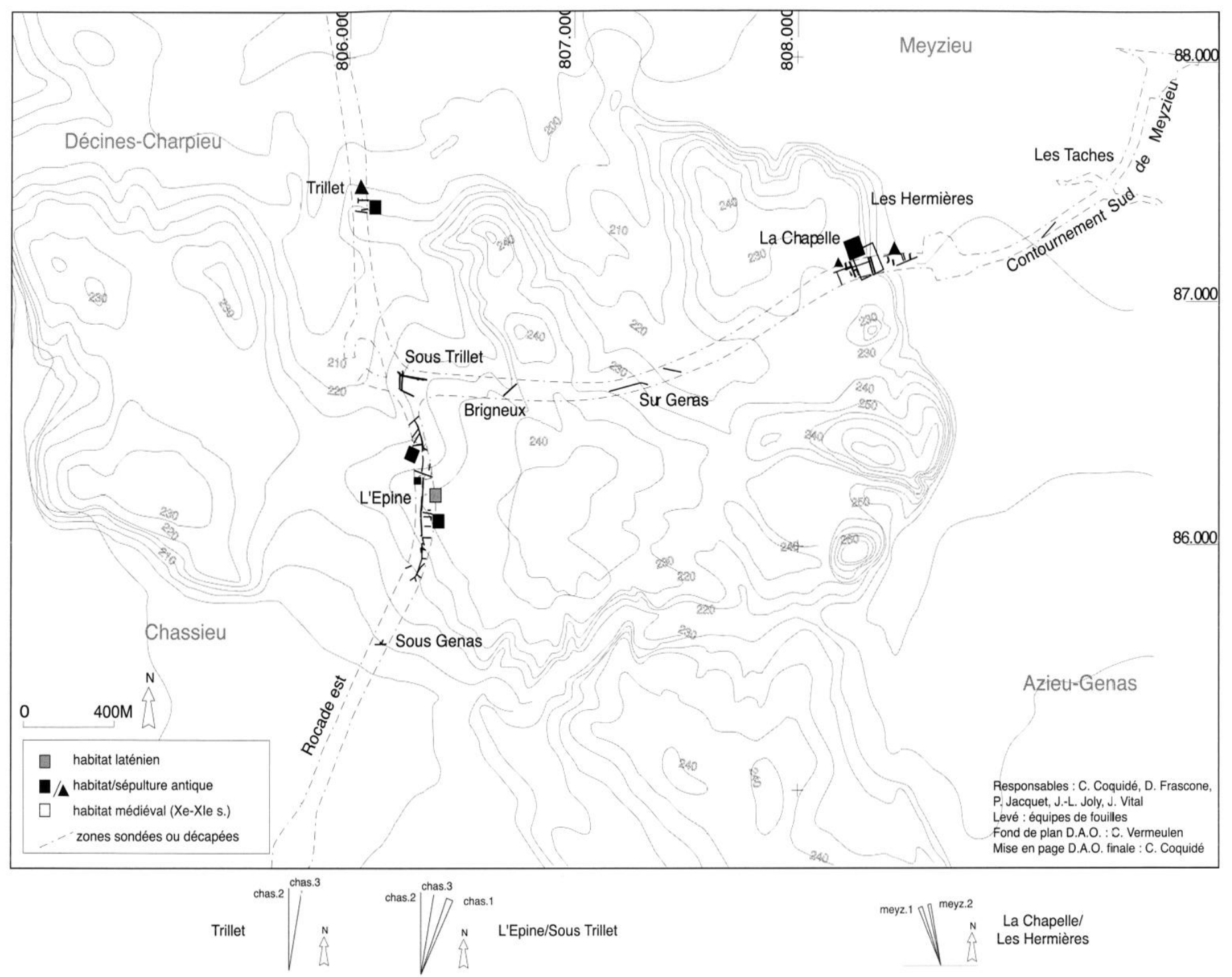

Fig. 3. Fossés, vestiges historiques et topographie autour de la butte de Chassieu-Genas-Décines-Meyzieu (zone d'étude I).

de placages de loess pouvant atteindre plusieurs mètres d'épaisseur alors que diverses colluvions limoneuses ont empâté ses reliefs au cours des siècles. En contrebas, la plaine fluvio-glaciaire présente un sous-sol sablo-graveleux où seuls quelques microreliefs cachent encore un dépôt loessique ou colluvié.

Au cours de la Protohistoire, les implantations humaines sont marginales (ou érodées) ne marquant aucune préférence sitologique et occupent l'ensemble du relief. Plus tard, des occupations antiques, parfois vastes et originales ${ }^{3}$, semblent s'orienter vers le rebord de la butte alors que quelques villae préfèrent la plaine au pied des pentes. Le Moyen Age voit le regroupement progressif vers les centres habités actuels, le site de Meyzieu "la Chapelle" étant l'un des jalons intermédiaires, occupé dès l'Antiquité puis abandonné vers le XII ${ }^{\mathrm{e}}$ s.. Les quatre communes actuelles (Chassieu, Décines, Meyzieu et Genas) ont prolongé cette tendance, englobant des centres anciens mitoyens (Charpieu à Décines, Azieu à Genas....) (Monnier 1990).

L'étude met en relief 5 groupes d'orientations dont deux se retrouvent sur plusieurs sites laténiens, antiques ou médiévaux ${ }^{4}$.

3 Chassieu "l'Epine" (Coquidé, Vermeulen 1999) ou Meyzieu "Trillet" sont en effet des établissements ruraux difficiles à interpréter. Ni vicus, ni villa, les occupations antiques mises au jour sont encore très imprégnées par les traditions dites indigènes de par leur organisation spatiale et leur mobilier.

4 Certains tracés ne s'intègrent pas à cette étude. Il s'agit de longs tracés totalement incurvés, peut-être de vastes enclos protohistoriques (à Chassieu "L'Epine" et à Meyzieu "Sur Genas"). 


\section{Fossés, habitats et indices de datation}

A l'est de la butte, sur les sites mitoyens de Meyzieu "La Chapelle" situé en haut de versant est et "les Hermières" en contrebas, au pied de la pente, on observe deux groupes d'orientations (Coquidé, Faure-Boucharlat, Forrest 2001, 284 et 285 ; Coquidé 1997, 22).

- Meyzieu 1 (or. de 19 à $23^{\circ}$ Ouest) : l'orientation la plus prégnante est matérialisée par une suite de fossés parallèles imbriqués dans une occupation antique qui se développe surtout hors de l'emprise de la fouille. Une certaine régularité dans l'implantation des tracés, tous les 68 à $85 \mathrm{~m}$, laisse l'impression d'un espace quadrillé.

Sur le haut de versant, on note trois fossés de largeur variable (de 0,95 à $2 \mathrm{~m}$ ), dont l'un comporte un accès menant à des bâtiments du $\mathrm{IV}^{\mathrm{e}} \mathrm{s}$. et un autre à une extension sous forme d'enclos ayant livré des fragments de céramiques communes claires. Plus loin, sur la pente, un fossé palissade présentant un blocage de galets et de tuiles $s$ 'incurve vers une carrière d'extraction des $\mathrm{II}^{\mathrm{e}}$-III ${ }^{\mathrm{e}} \mathrm{s}$. alors qu'un dernier fossé situé dans la plaine est bordé de quelques incinérations contemporaines à la carrière. L'occupation médiévale qui suivra reprendra cette inclinaison.

- Meyzieu 2 (or. de $10^{\circ}$ à $13^{\circ}$ Ouest) : le second groupe d'orientations est surtout relevé sur les "Hermières" avec deux axes perpendiculaires ; un seul fossé s'y rattache sur "La Chapelle".

Au sud de la butte morainique à Chassieu "L'Epine" et à "Sous Trillet", ces deux chantiers ne reflétant qu'un seul site, il existe 3 groupes d'orientations dont deux sont bien représentés. (Coquidé, Vermeulen 1999, 206 et 207)

- Chassieu 1 (or. de 20 à $25^{\circ}$ Est) : les fossés relevant de cette orientation s'imbriquent dans l'habitat du Ier $s$. de notre ère et se développent vers le nord sur les deux sites. On remarque dans les espaces intercalaires des fossés présentant des variations progressives en éventail (de $30^{\circ}$, $40^{\circ}$, jusqu'à $50^{\circ}$ Est).

- Chassieu 2 (or. approx. Nord) : une seconde orientation, composée de fossés dont les retours d'angle présentent souvent des tracés curvilignes, est globalement nordsud. Cette inclinaison correspond à l'axe de l'occupation laténienne puis à celle de l'un des habitats antiques. Un peu de matériel céramique s'y associe.

- Chassieu 3 (or. perpendiculaire à $10^{\circ}$ Est) : une troisième orientation n'apparaît qu'à "Sous Trillet" et est associée à un fossé du groupe Chassieu 2. Une analyse $14 \mathrm{C}$ de charbons de bois issus d'un foyer installé dans l'un des comblements du fossé indique une période de fonctionnement située entre le $\mathrm{II}^{\mathrm{e}} \mathrm{s}$. et le $\mathrm{VI}^{\mathrm{e}} \mathrm{s} .{ }^{5}$.

Au nord de la butte, à Meyzieu "Trillet", deux orientations déjà mentionnées sont reconnues (Blaizot 2001, 277 279).

- Chassieu 2 (or. nord à $3^{\circ}$ Ouest) : on remarque d'abord un fossé formant un angle avec une déviance à $7^{\circ}$ Ouest, qui livre un matériel céramique du $\mathrm{I}^{\mathrm{er}} \mathrm{s}$.. Cet ensemble est en partie contemporain des excavations aménagées qui lui sont mitoyennes.

- Chassieu 3 (or. $10^{\circ}$ Est) : un fossé s'individualise au sud et a livré quelques tessons du $\mathrm{V}^{\mathrm{e}} \mathrm{s}$. Cette inclinaison se reflète également dans l'axe global de la nécropole située au nord et datée de la seconde moitié du IV $\mathrm{e} s$. au plus tôt et s'étendant peut-être jusqu'au VII/VIII ${ }^{e} s$.

D'autres décapages ont livré des fossés qui semblent isolés de tout contexte :

- A Meyzieu "Sur Genas", un très large fossé (1. : $3 \mathrm{~m})$ reprend l'axe de $10^{\circ}$ Est (Chassieu 3).

-A Chassieu "Brigneux" on enregistre un tracé orienté à $40^{\circ}$ Est.

\subsection{De très larges décapages sur un versant sud de la butte morainique de Saint-Priest (zone d'étude 2)}

Les études archéologiques de la Zac des Perches, puis de celles des Feuillis nord et sud et du Boulevard Urbain Est ont permis le sondage de 87 hectares et la fouille de presque 14 hectares. A l'inverse des deux tracés présentés précédemment, si ces ouvertures transmettent un aperçu du relief beaucoup moins transversal, elles offrent en revanche une vision extensive d'un même secteur ${ }^{6}$ (fig. 4).

La butte morainique autour de laquelle s'articulent ces décapages est de forme oblongue et s'étend du nord-ouest au sud-est, de la commune de Bron à celles de Saint Laurent de Mure et de Grenay. La ville de Saint-Priest s'est installée sur le versant sud de la partie médiane de ce relief.

Les vestiges qui se situaient dans une zone agricole au nord de la commune, entre 204 et 207 m d'altitude, occupaient un relief peu accentué, atténué par l'érosion et par d'épais dépôts loessiques. En contrebas, la concavité de la plaine a favorisé l'accumulation de colluvions protectrices et généré la formation d'une stratigraphie complexe.

Les principaux sites mis au jour sont d'époque protohistorique et n'ont livré aucun fossé associé (Hénon 1999). Ces vestiges n'ont pas été représentés sur les figures. En revanche, de nombreux fossés d'époque historique sont apparus aux Feuillis et sur le "BUE" alors que 


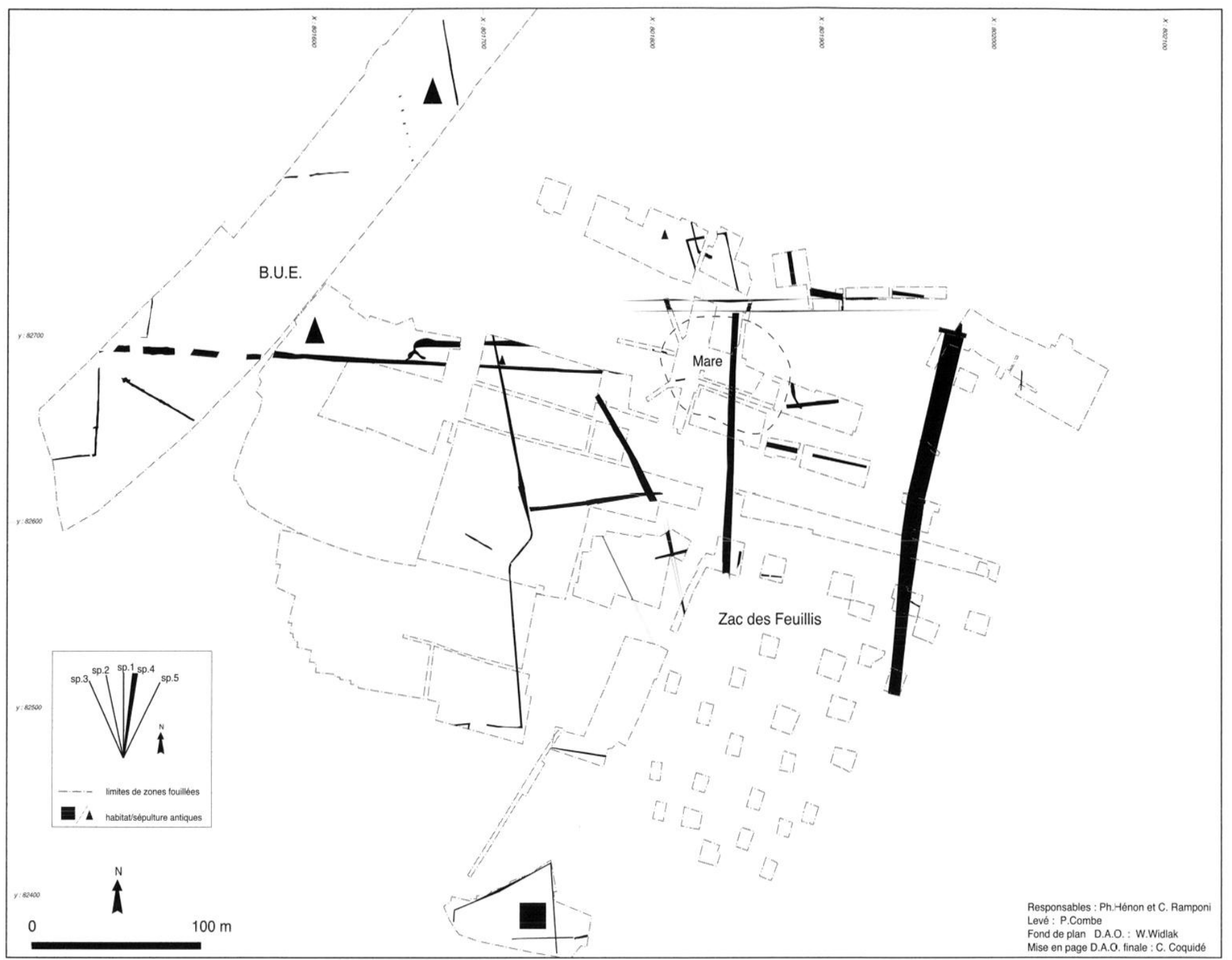

Fig. 4. Fossés et vestiges historiques sur le versant sud de la butte morainique de Saint-Priest "les Feuillis" et "BUE" (zone d'étude 2).

le site des Perches, mitoyen au nord, n'a révélé que très peu de structures de ce type.

On note plus de 80 tracés de portées inégales qui sont, apparemment, les principaux témoins de l'occupation des lieux. Les autres implantations restent marginales : un bâtiment excavé et enclos d'époque antique au sud, une large mare (également antique ?) au centre et plusieurs petits ensembles sépulcraux au nord.

On relève 5 groupes d'inclinaisons dont deux sont bien représentés.

\section{Fossés et occupations marginales}

Ces décapages aux très larges emprises ont permis de prendre du recul et de percevoir les tracés dans leur ensemble. La plupart des fossés sont strictement linéaires alors que d'autres forment des angles associant parfois des groupes d'orientations divergents, certaines sections s'incurvant même sur de longues distances.
- Saint-Priest 1 (or. : Nord) : le décapage en aire ouverte a permis le repérage de nombreux tracés. Parmi ceux-ci, de larges fossés parallèles reconnus sur toute la largeur des décapages témoignent sans doute du passage d'anciens chemins. Un certain nombre de fossés sont orientés au nord, mais avec de légères variantes. Cette orientation est également celle du secteur antique situé aux marges du décapage vers le sud. On y observe de larges excavations aménagées qui sont bordées d'un enclos partiellement isocline. Ses profils évoluent rapidement d'un comblement drainant essentiellement composé de galets à un remplissage à dominante limoneuse puis à un profil palissadé. Bâti isolé ou excentré à proximité d'une occupation plus large vers le sud, son contexte reste inexploré.

- Saint-Priest $2\left(13^{\circ}\right.$ Ouest $)$ : cette seconde orientation se signale par une série de tracés parallèles ou perpendiculaires au nord-est des décapages. Dans ce secteur, la mare aménagée de $4000 \mathrm{~m}^{2}$ supposée antique est antérieure aux fossés de ce groupe. 
Au centre de l'emprise, on remarque également un axe dont l'orientation subit un décalage latéral sur sa partie médiane. Sur son extrémité nord, une série de petites fosses à galets double ce tracé sur une quarantaine de mètres. Au sud, cel axe se connecte avec un fossé associé au groupe de Saint-Priest 1.

D'autres inclinaisons mineures sont relevées ; seuls les vestiges conservant plusieurs dizaines de mètres linéaires sont mentionnés :

- Saint-Priest 3 (or. $26^{\circ}$ Ouest) : se connecte à l'enclos antique sud du groupe Saint-Priest 1 (2 occurrences).

- Saint-Priest 4 (or. $7 / 10^{\circ}$ Est) : 5 occurrences.

- Saint-Priest 5 (or. $28^{\circ}$ Est) : 3 occurrences.

\section{La lecture des données de fouille}

La synthèse des données issues de ces deux zones d'études permet quelques remarques concernant la chronologie, la fréquence des inclinaisons, l'influence de la topographie ou l'imbrication des ensembles de fossés ou de fosses à galets dans les habitats. Sur certains de ces thèmes, l'enquête a été élargie aux autres fouilles de l'Est lyonnais.

\subsection{De rares pistes chronologiques}

Les indications chronologiques disponibles que ce soit sur chaque zone d'étude ou sur la totalité du secteur abordé ne sont qu'une compilation de péripéties locales qui ne peuvent donner lieu à aucune généralisation. Par large période chronologique, elles semblent toutefois s'accorder sur des présomptions de présence/absence.

Elles s'appuient aussi bien sur des chronologies relatives telles que l'imbrication des fossés avec les occupations humaines ou des chronologies absolues basées sur des ensembles céramiques ou une datation $\mathrm{C} 14$ (fig. 5a). Malgré cela, un nombre non négligeable de tracés reste non daté et la lecture des intersections est souvent impossible à cause du manque de profondeur des vestiges.

A la lecture des plans de masse, peu de tracés protohistoriques ont été mis au jour. Hormis de longs axes curvilignes et très érodés sur Chassieu "l'Epine" qui pourraient se rapprocher de larges enclos agraires, seuls quelques enclos sont attribués à La Tène finale (Chassieu "l'Epine" ou Beligneux "Camp de Chânes"). C'est à partir de cette période que l'on observe l'émergence des tra- cés linéaires, confirmée par leur multiplication durant l'Antiquité (fig. 5).

D'une manière plus précise, les ensembles orientés nord-sud et de 20 à $25^{\circ}$ Est apparaissent très tôt (dès le Ir s. ap. J-C.). A Chassieu "l'Epine", les bâtiments laténiens adoptent d'ailleurs déjà une orientation nord-sud dont l'Antiquité ne fait peut-être qu'hériter. Ailleurs, les inclinaisons allant de 19 à $23^{\circ}$ Ouest et celle de $10^{\circ}$ Est sont attestées à la charnière des Haut et Bas Empire. Toutes perdurent au Bas-Empire (jusqu'au III' $\mathrm{s}$. au moins à "Trillet", "L'Epine" ou "La Chapelle «). L'inclinaison de $10^{\circ}$ Est est observée depuis l'Antiquité et fonctionne encore entre les $\mathrm{V}^{\mathrm{e}}$ et $\mathrm{VIII}^{\mathrm{e}} \mathrm{s}$. $\left(\mathrm{C}^{14}\right.$ et céramiques à "Sous Trillet" et céramiques à "Trillet"). A Saint-Priest, la seule orientation clairement associée à un habitat antique est orientée au nord.

Plus tard, malgré un panel de sites médiévaux non négligeable, seuls les sites du $\mathrm{X}^{\mathrm{e}}-\mathrm{XI}^{\mathrm{e}}$ s. de Communay "Charvas" ainsi que celui de "la Chapelle" livrent un réseau fossoyé. Sur ce dernier site, un axe orienté de 19 à $23^{\circ}$ Ouest semble être contemporain de l'habitat des $X^{\mathrm{e}}$ $\mathrm{XI}^{\mathrm{e}}$ s. tout en oblitérant la trame quadrillée antique.

La quasi-absence de tracé linéaire rattachables aux périodes protohistoriques témoigne sans doute de l'évolution des marquages au sol et de la disparition de traces telles que les haies, les bornes lithiques et végétales ou les empierrements de surface. Par la suite, si le fossé devient d'un usage commun depuis le Ir $^{\text {r }}$ s. av. J.-C. jusqu'à la fin de l'Antiquité, son utilisation ne s'associe plus que rarement aux sites médiévaux des $\mathrm{X}^{\mathrm{e}}$ et $\mathrm{XI}^{\mathrm{e}}$ s.. La période intermédiaire du Haut Moyen Age, encore sous-documentée, ne permet aucune observation archéologique de cette rupture des usages dont l'évolution est pour l'instant impossible à cerner (Faure-Boucharlat 2001, 282-325). Les tracés mis au jour à l'écart des occupations continuent à poser problème et restent non datés.

\subsection{Des orientations récurrentes}

En élargissant l'étude à d'autres sites est lyonnais, on observe un très large éventail d'orientations (fig. $5 a$ et $b)^{7}$. A l'évidence, un même site révèle plusieurs inclinaisons parfois totalement divergentes (lecture verticale - fig. 5b). Ce graphique, qui inclut les zones d'études exposées précédemment, souligne l'influence de la valeur proche du nord/sud (lecture horizontale) ${ }^{8}$.

7 La difficulté réside dans la restitution de l'inclinaison d'une structure qui, sur un même chantier, peut varier selon le tronçon de fossé mis au jour ; un même vestige oscille fréquemment de quelques degrés sur quelques dizaines de mètres de distance.

8 Le traitement en un seul tableau de zones largement décapées, favorisant la mention d'inclinaisons identiques puisque issues d'un contexte proche, et de sites plus isolés ne paraît pas fausser les résultats : la variété des orientations est respectée et des occurrences d'axes identiques interviennent sur des secteurs distants. 


\begin{tabular}{|c|c|c|c|c|}
\hline SITES & $\mathrm{n}^{\circ}$ site & Orientation & Indices chrono. & Notes \\
\hline Beynost "Grande Croix" & 1 & -32 & Gallo-romain & \\
\hline Beligneux "Camp de Chânes" & 2 & -40 & La Tène finale & \\
\hline \multirow[t]{2}{*}{ Chassieu "L'Epine" } & 3 & $0 / 3$ & La Tène F./GR & Chass. 2 \\
\hline & 3 & $20(30 / 40)$ & Gallo-romain & Chass. 1 dont chemin \\
\hline \multirow[t]{3}{*}{ Chassieu "Sous Trillet" } & 4 & 0 & & Chass. 2 associé Chass. 1 \\
\hline & 4 & 10 & Gallo-romain & Chass. 3 (14C : IIe/VIe s.) \\
\hline & 4 & 25 & & Chass. 1 dont chemin \\
\hline Communay "Charvas" & 5 & $25 / 30$ & Moyen-Age & Xe-XIe s. \\
\hline Corbas "B.U.S. Grand Champ" & 6 & -30 & & carrefour \\
\hline Corbas "Corbèges" & 7 & -7 & & \\
\hline \multirow[t]{3}{*}{ Feyzin "Champ Plantier III" } & 8 & $0 /-3$ & Gallo-romain & \\
\hline & 8 & -34 & & \\
\hline & 8 & 25 & & \\
\hline Janneyrias "le Viere-la Plaine"/A432 & 9 & 10 & Gallo-romain & \\
\hline Marennes "Le Pillon" & 10 & $5 / 6$ & Gallo-romain & \\
\hline \multirow[t]{2}{*}{ Meyzieu "Trillet" } & 11 & $0 /-3$ & Gallo-romain & Chass. 2 \\
\hline & 11 & 10 & Gallo-romain & Chass. 3 (TPQ Ve s.) \\
\hline \multirow[t]{2}{*}{ Meyzieu "La Chapelle" } & 12 & $-19 /-23$ & GR/M.-Age & Meyz. 1 (Ie-IVe s. et Xe-XIe s.) \\
\hline & 12 & $-10 /-13$ & & Meyz. 2 \\
\hline \multirow[t]{2}{*}{ Meyzieu "Les Hermières" } & 13 & -10 & & Meyz. 2 \\
\hline & 13 & -20 & Gallo-romain & Meyz. 1 \\
\hline Meyzieu "La Dent" & 14 & 20 & Gallo-romain & \\
\hline Mions "Pierres Blanches" & 15 & -10 & & \\
\hline Pusignan "Vandessine"/A432 & 16 & 12 & & \\
\hline St Priest "Ilford" & 17 & $-2 /-3$ & Gallo-romain? & chemin \\
\hline \multirow[t]{2}{*}{ St Priest "Les Perches" } & 18 & 0 & & \\
\hline & 18 & 38 & & \\
\hline \multirow[t]{2}{*}{ St Priest "Réservoir" } & 19 & -7 & Gallo-romain? & \\
\hline & 19 & $13 / 15$ & & \\
\hline \multirow[t]{5}{*}{ Saint Priest "ZAC des Feuillis" et "B.U.E." } & 20 & $0 / 3$ & Gallo-romain & SP 1 dont chemins \\
\hline & 20 & $-11 /-13$ & & SP 2 associé SP 1 \\
\hline & 20 & -26 & & SP 3 associé SP 1 \\
\hline & 20 & $7 / 10$ & & SP 4 \\
\hline & 20 & 28 & & SP 5 \\
\hline St Rom. de Jal. "Sambettes" & 21 & -15 & & \\
\hline St Rom. de Jal. "Vernai" & 22 & -30 & Gallo-romain & \\
\hline Vénissieux "Vieux Bourg" & 23 & $-15 /-20$ & Gallo-romain & \\
\hline Vill. d'Anthon "Com. de Charvas"/A432 & 24 & $-40 /-30 /-21 /-11 /-8 /-2$ & & \\
\hline Vill. d'Anthon "Com. d'Arrézieux" & 25 & $10 / 12$ & Gallo-romain & \\
\hline
\end{tabular}

Fig. 5 a et b. Tableau et graphique des inclinaisons relevées sur un panel de sites de l'Est lyonnais.

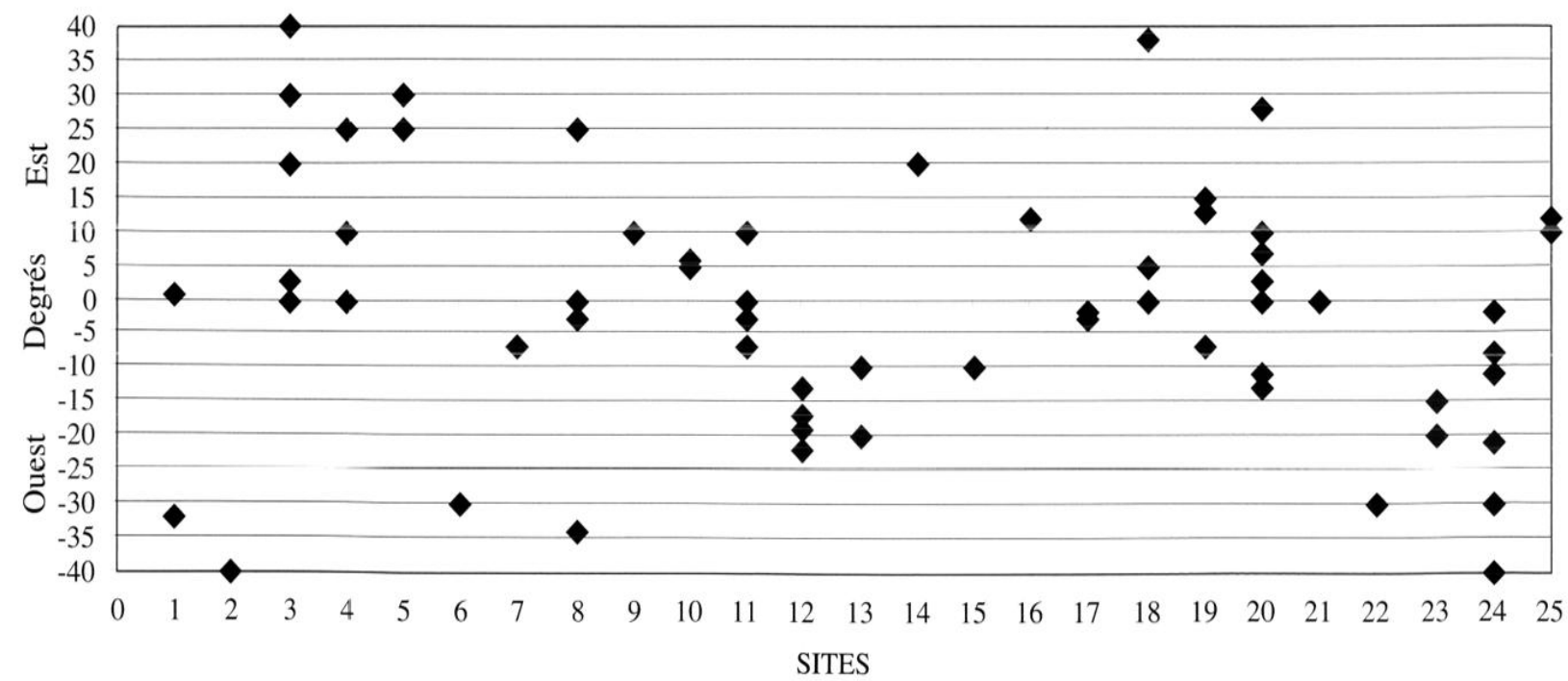




\begin{tabular}{|c|l|l|l|l|}
\hline & Groupes & \multicolumn{1}{|c|}{ Orientations } & Indic. chrono. & \multicolumn{1}{|c|}{ Zones d'influence } \\
\hline ZONE 1 & Meyzieu 1 & $19 / 23^{\circ} \mathrm{O}$ & Gallo-romain & bien implantée sur le site \\
\hline & Meyzieu 2 & $10 / 13^{\circ} \mathrm{O}$ & & zones 1 et 2 \\
\hline & Chassieu 1 & $20 / 25^{\circ} \mathrm{E}$ & Gallo-romain & bien implantée sur le site \\
\hline & Chassieu 2 & Or. au Nord (approx.) & Gallo-romain & très présente, zones 1 et 2 \\
\hline & Chassieu 3 & $10^{\circ} \mathrm{E}$ & GR/post IIe-Ve s. & zones 1 et 2 \\
\hline & Brigneux & $40^{\circ} \mathrm{E}$ & & fossé isolé \\
\hline & & & & \\
\hline ZONE 2 & Saint Priest 1 & Or. au Nord (approx.) & Gallo-romain & très présente, zones 1 et 2 \\
\hline & Saint Priest 2 & $11 / 13^{\circ} \mathrm{O}$ & & zones 1 et 2 \\
\hline & Saint Priest 3 & $26^{\circ} \mathrm{O}$ & & site \\
\hline & Saint Priest 4 & $7 / 10^{\circ} \mathrm{E}$ & & zones 1 et 2 (10 ${ }^{\circ}$ E) \\
\hline & Saint Priest 5 & $28^{\circ} \mathrm{E}$ & & site \\
\hline Notes & & & & en l'état des décapages \\
\hline
\end{tabular}

Fig. 6. Tableau récapitulatif des orientations observées sur les deux zones d'étude.
Certaines orientations sont enregistrées sur les deux zones d'étude ainsi que sur des sites éloignés. Ainsi, l'orientation globalement nord-sud est dominante et se retrouve sur les sites de la Rocade est ("L'Epine", "Sous Trillet", "Trillet" et "Sous Genas") et à Saint-Priest sur "les Feuillis" ou Feyzin "Champ Plantier III" et Villette d'Anthon "Communaux d'Arézieux" (fig. 5b et 6).

L'inclinaison de $10 / 13^{\circ}$ Ouest est prépondérante aux "Feuillis" (zone d'étude 2) mais on la remarque également sur plusieurs sites de la zone 1 à "la Chapelle" et aux "Hermières" ou à Mions "Pierres Blanches". Les valeurs d'inclinaisons entre 7 et $10^{\circ}$ Ouest sont également remarquées du sud (Vénissieux "Vieux Bourg") au nord de la région étudiée (Meyzieu "Trillet"). De même, l'orientation $10^{\circ}$ Est qui est la seconde occurrence en nombre de tracés sur la première étude de cas, est notée à "Sous Trillet", "Sur Genas", et "Trillet" mais elle se remarque également à Saint-Priest où elle demeure néanmoins peu fréquente (fig. 6). Elle apparaît sur d'autres communes nord-orientales comme Villette d'Anthon ou Janneyrias.

On note deux à trois occurrences pour des inclinaisons proches des 20,25 et $30^{\circ}$ Est ou les 20,30 et $40^{\circ}$ Ouest. Les autres références se cantonnent à un seul site.

Ces observations individualisant chaque orientation ne doivent pas masquer les connexions qui existent entre elles. On a ainsi observé un lien entre Chassieu 1 et 2 à "l'Epine" à partir d'une même intersection qui semble résulter de la surimposition de deux réseaux distincts. Entre Saint-Priest 1 et 2 ou Saint-Priest 1 et Saint-Priest 3 en revanche, ce sont deux larges enclos aux branches divergentes qui se sont formés.

\subsection{Inclinaisons et topographie}

La superposition des fossés avec les courbes de niveau révèle d'évidents échos alors que d'autres tracés s'affranchissent nettement de leur environnement topographique (fig. 3).
- A "la Chapelle" et "Les Hermières", l'inclinaison de 17 à $22^{\circ}$ Ouest s'appuie sur l'orientation de la rupture de pente de la butte vers la plaine ${ }^{9}$. La seconde orientation, autour des $10^{\circ}$ Ouest, pourrait aussi être lue comme une variante de cette topographie locale, par exemple au nord du site.

- A "L'Epine" et à "Sous Trillet", l'orientation se développant en éventail à partir des $20^{\circ}$ Est, semble trouver un écho dans les auréoles du vallonnement, et par conséquent dans les ruissellements de pente, qui pourrait expliquer l'inclinaison de cet axe vers l'est tout au long du décapage.

-Aux "Feuillis", le constat est moins net. Deux orientations minoritaires pourraient s'appuyer sur la topographie, se plaçant soit parallèlement, soit perpendiculairement à des courbes locales (non illustré) : $26^{\circ}$ Ouest et 28 Est.

Ces observations sont facilitées sur des sites ayant bénéficié de larges décapages dans un environnement au relief marqué. A ce titre, la butte morainique de la zone d'étude 1, quadrillée de deux tracés soumis aux études d'impact archéologique, l'un nord-sud et l'autre est-ouest, s'est révélée une zone particulièrement significative. Mais là comme ailleurs, certaines orientations n'ont montré aucun assujettissement au relief. Parmi celles-ci, se distingue encore l'inclinaison orientée au nord qui ne trouve aucun écho ni sur ce relief ni sur la plaine dont les courbes décroissent d'Est en Ouest selon une inclinaison proche des $40^{\circ}$ Est (fig. 2).

\subsection{Traces et implantations humaines}

Sur la zone 1, les fossés et les habitats semblent aller de pair. En effet, les surfaces sondées ou décapées entre les occupations n'ont livré que peu de fossés et là où les sites sont rares, les fossés deviennent erratiques. De même, dans la plaine où peu de sites sont mis au jour, peu 


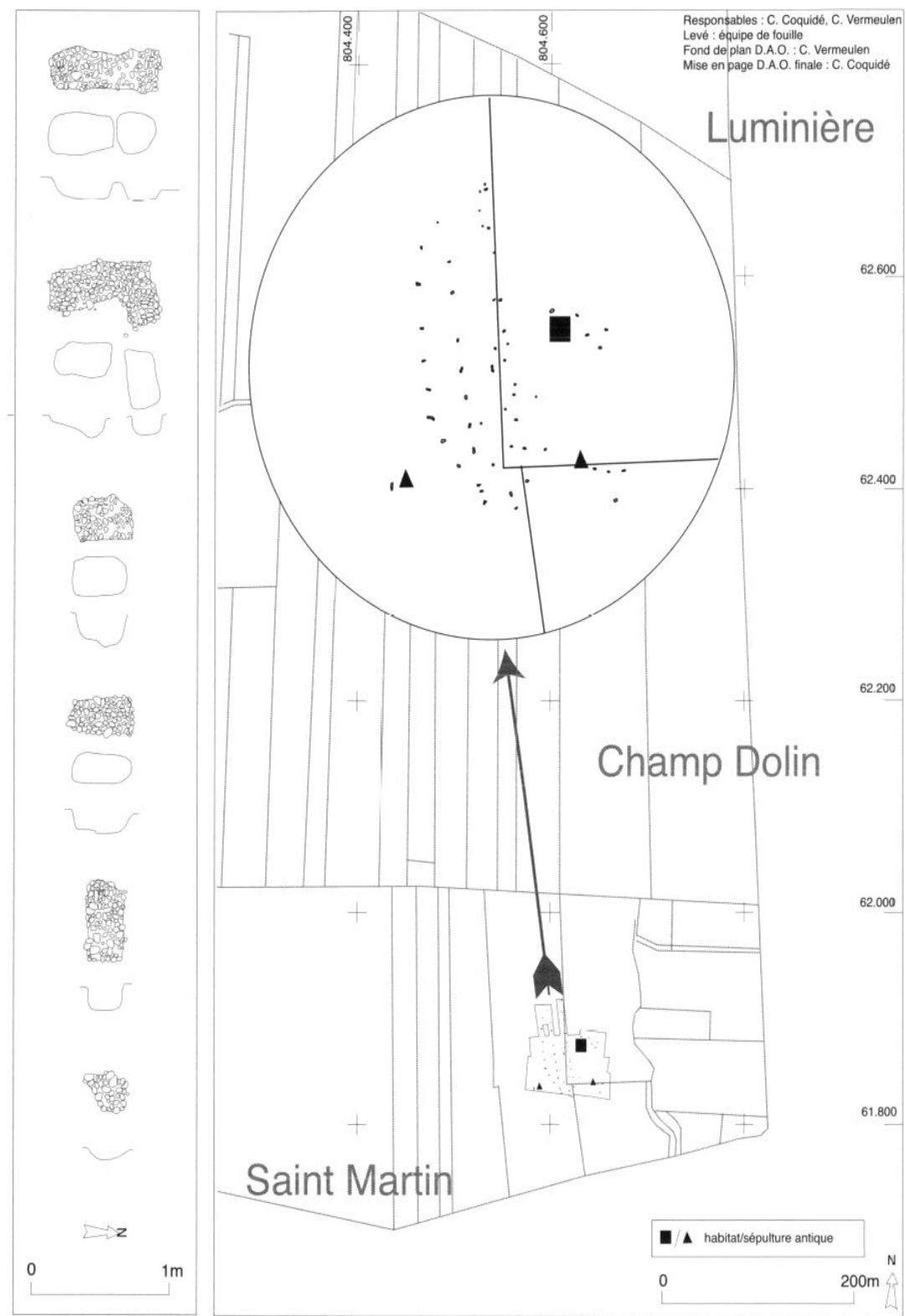

Fig. 7. Alignements de fosses à galets et cadastre napoléonien à Saint-Priest "Champ Dolin". Exemples de plans et de coupes.

de fossés sont apparus. Cette répartition différentielle illustre le rôle fédérateur des occupations humaines qui semblent être le point d'origine du développement des fossés (fig. 3). Deux schémas s'opposent : soit l'habitat sert de point d'origine pour le déploiement des fossés vers les marges de l'occupation comme à "l'Epine", soit il s'insère dans une trame assez régulière comme à " $\mathrm{La}$ Chapelle" et "Les Hermières".

Dans la zone 2 à Saint-Priest "les Feuillis" et "BUE", le seul habitat observé au sud du décapage génère son propre enclos alors que les autres fossés ont été mis au jour sans qu'aucune occupation ne soit associée. Pourtant, une mare aménagée de $4000 \mathrm{~m}^{2}$ et quelques tegulae confirment la proximité des implantations humaines. Plus au nord et bien qu'il s'agisse d'un secteur mitoyen, la "Zac des Perches" (non illustrée) ne livre plus que de très rares fossés malgré plusieurs hectares décapés, révélant de nouveau de larges zones apparemment non délimitées (fig. 4).

Les fossés hors de tout contexte, à l'écart de toute occupation, semblent peu nombreux. On note pourtant quelques tracés erratiques à Chassieu "Sur Genas", Mions "Pierres Blanches" ou Corbas "Corbèges". De profils prononcés, ces structures aux tracés linéaires sont suivies sur de grandes distances et semblent marquer la limite d'un territoire, quel qu'il soit. Autre vestige répondant à une fonction qui dépasse l'échelle strictement locale, les traces du réseau viaire qui prennent souvent la forme de deux fossés parallèles comme à Saint-Priest "Illford" où ils matérialisent une voie probablement antique.

\subsection{Des structures originales : les lignes de fosses à galets}

Parmi la trame des fossés, des ensembles de fosses remplies de galets attirent l'attention à Saint-Priest "les Feuillis", à Meyzieu "la Chapelle" et à Meyzieu "les Taches". Leur disposition est rarement aléatoire : elles suivent un même faisceau linéaire ou forment des angles. Ces fosses sont de plans et de modules variables, non aménagées. Les plus petites sont de simples amas de galets, d'aspects circulaires ou informes ; plus importantes, elles adoptent une forme rectangulaire à fond souvent plat mais irrégulier. Quelques exceptionnels plans en "V" ont été mis au jour (fig. 7, 8 et 9).

Leur comblement est uniquement constitué de galets non fracturés et le limon ne remplit que rarement les interstices. Les seules autres, et rares, inclusions recueillies sont des fragments de tuiles indéterminées ou de terre cuite. Ces fosses restent difficiles à dater car elles ne s'associent pas aux occupations humaines et leur usage a disparu des pratiques agraires contemporaines. Une seule série diverge de ce schéma : des amas de galets observés parallèlement à un axe médiéval et installés dans une batterie de foyers (Meyzieu "la Chapelle").

\section{Les hypothèses à rejeter}

La particularité de ces vestiges a donné lieu à diverses lectures. L'épierrage est la première de ces hypothèses. Quand de telles fosses existent, elles sont de tailles conséquentes, aptes à accueillir les innombrables galets de la moraine. Ici, les fosses sont de modules beaucoup plus 


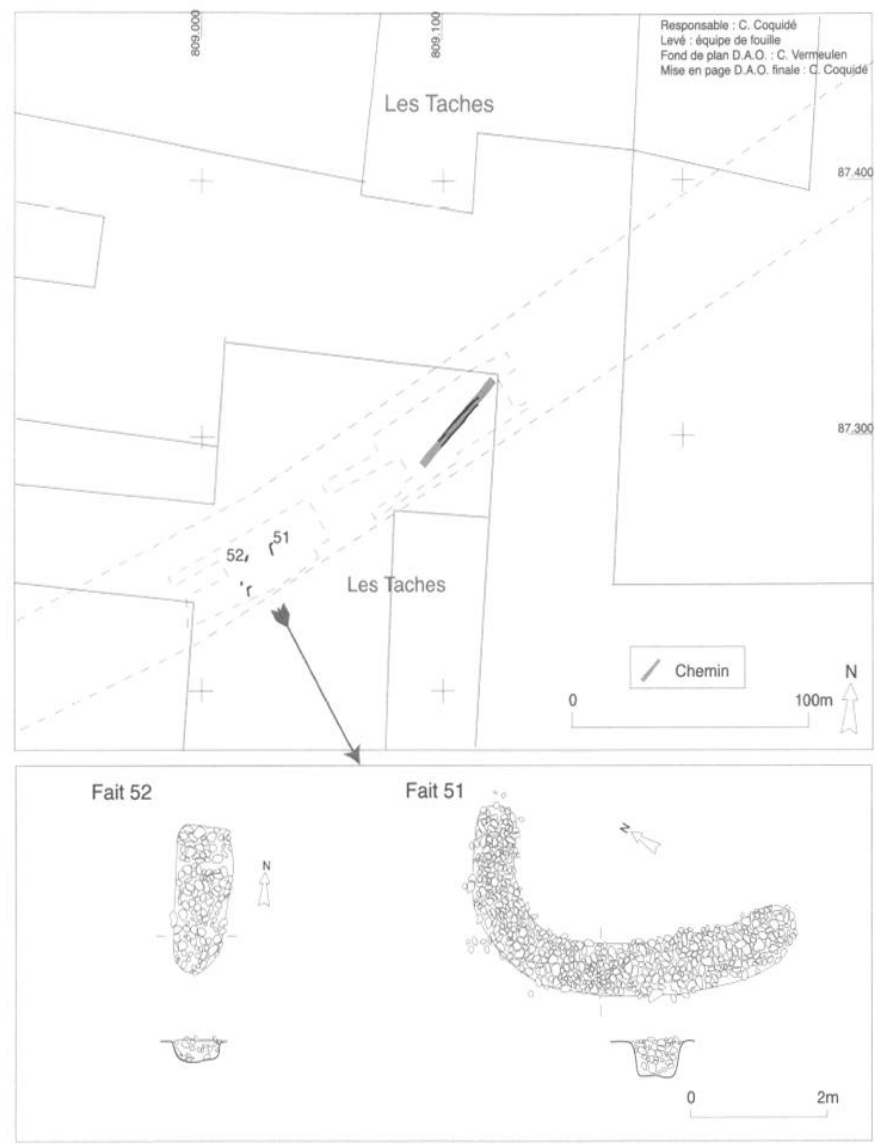

Fig. 8. Fosses à galets à Meyzieu "les Taches".

modestes, voire inopérants pour la fonction. Elles sont même parfois installées sur des sols limoneux où les galets sont absents. Leur relation avec un mode de culture particulier, la vigne, est également proposée, sans fondement jusqu'à présent. Il ne s'agit pas plus de calages, aucune trace de négatif de poteau n'étant jamais observée et les comblements restant identiques quel que soit la structure ou le site. Au regard des plans adoptés (écartement des vestiges entre eux, alignements sur des dizaines de mètres), le solin de bâti semble hors de propos.

\section{Des éléments de réponses}

Ces fosses semblent participer à un environnement rural isolé. De par l'absence systématique d'inclusion charbonneuse ou de fragment céramique, elles paraissent éloignées des habitats. Pour l'instant, seuls deux prélèvements palynologiques ont été effectués sur un fin niveau limono-sableux tapissant le fond de deux fosses à SaintPriest "Champ Dolin". Leurs résultats montrent un envi-

Fig. 9. Report des fossés sur le cadastre napoléonien à SaintPriest "les Feuillis" et "BUE” (zone d’étude 2).

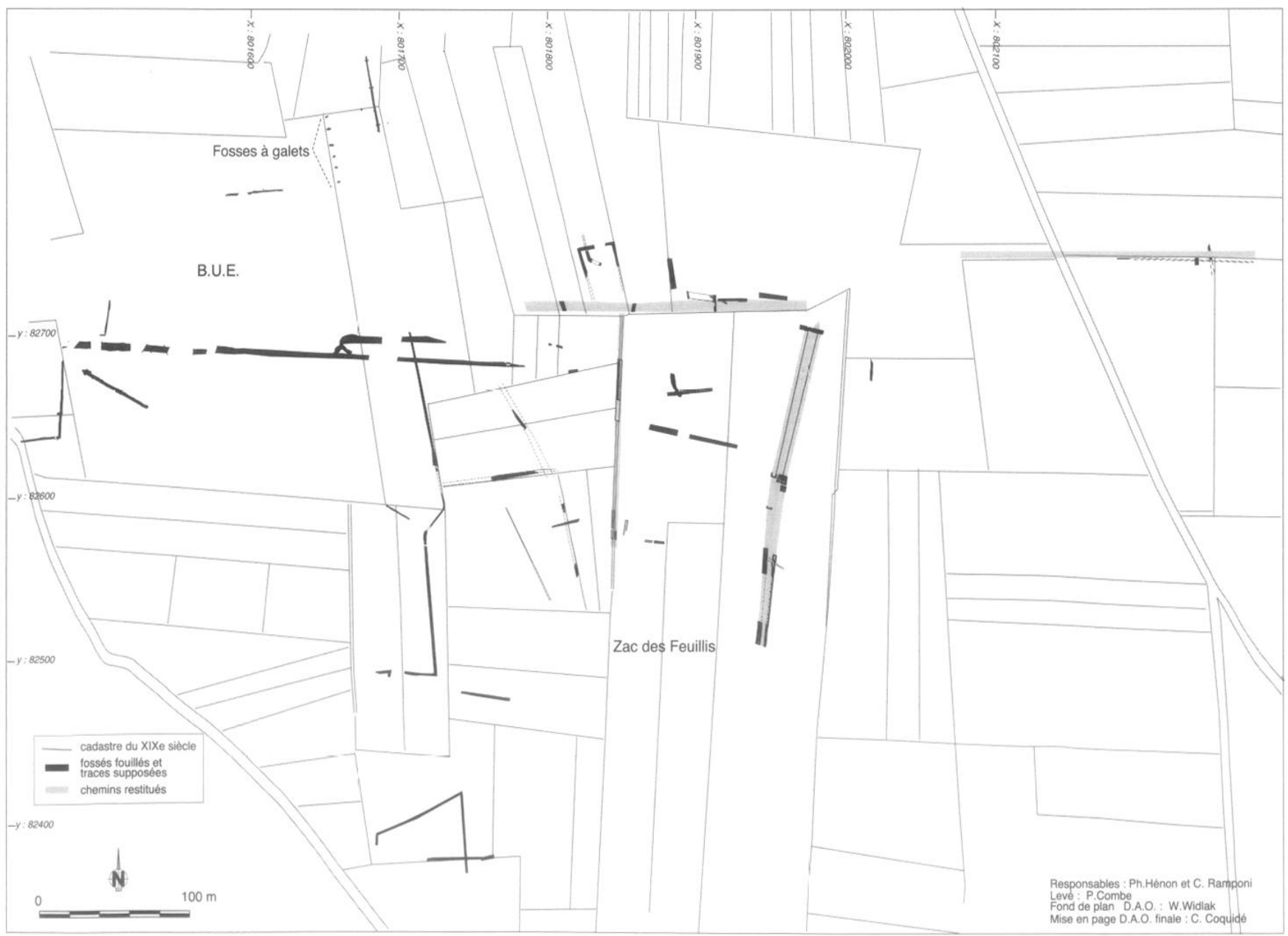


ronnement ouvert, un espace herbeux et la proximité d'une forêt ; aucune trace d'activité agricole n'est relevée ${ }^{9}$.

\section{Données archéologiques et cartographiques}

La confrontation des fonds carto- et photographiques avec les plans de masse des décapages de l'Est lyonnais, visions restreintes à quelques hectares mais livrant un écheveau de tracés beaucoup plus dense, apporte un éclairage complémentaire à l'étude.

Deux approches sont privilégiées. En premier lieu, les observations faites lors de la synthèse des données de terrain sont mises en regard des différentes hypothèses sur les parcellaires de l'Est lyonnais émises à partir des travaux de carto- et photo-interprétation. Puis, la superposition des plans de masse avec le cadastre napoléonien, premier plan parcellaire levé à l'échelle nationale vers la fin du premier tiers du $\mathrm{XIX}^{\mathrm{e}} \mathrm{s}$., permet de révéler les décalages et les survivances entre les traces enfouies et les limites de surface actuelles.

\subsection{L'évolution de la recherche sur les parcellaires en région lyonnaise}

Les premières études sur les parcellaires se sont d'abord tournées vers la recherche d'éventuelles centuriations, réseaux planifiés antiques dont la présence pouvait encore marquer l'actuelle division de l'espace.

Plusieurs hypothèses ont concerné l'Est lyonnais ; elles sont basées sur l'observation cartographique d'orientations dominantes dans le parcellaire actuel puis sur l'application d'une grille supposée standard de croisements d'axes tous les $710 \mathrm{~m}$ (Chouquer, Favory 1980 ; Chouquer, Favory 1991). Deux réseaux sont ainsi apparus, l'un ayant Vienne pour origine, l'autre développé à partir de Lyon :

$$
\begin{aligned}
& \text { Lyon }=23,3^{\circ} \text { Est } \\
& \text { Vienne }=0^{\circ}
\end{aligned}
$$

Notons que ces inclinaisons sont également le reflet de deux axes routiers majeurs dont les tracés sont contraints par la topographie : la percée vers les Alpes de la voie Lyon-Grenoble/Chambéry orientée autour des $25^{\circ}$ Est et respectant les couloirs des anciens écoulements glaciaires et la route nord-sud de Lyon à Vienne longeant la rive droite du Rhône (fig. 2).

Selon le graphique des occurrences (fig. 5b), l'orientation nord-sud apparaît fréquemment et s'associe volontiers à une occupation antique, l'inclinaison de $23,3^{\circ}$ Est n'est pas observée ; ses valeurs proches $\left(20\right.$ ou $25^{\circ}$ Est $)$ sont peu représentées.

Par la suite, ces premières hypothèses ont évolué. Technologie numérique à l'appui, les études ont pris en compte la notion de "périodicité" de ces limites agraires ; des systèmes informatiques permettent d'en calculer les occurrences, c'est à dire de repérer la répétition d'une même orientation selon une valeur métrique inter axe équivalente à l'actus ou à ses divisions. La présence d'une orientation dominante associée à des occurrences significatives peut alors indiquer l'existence d'une centuriation. Néanmoins, d'autres formes parcellaires organisées, médiévales et modernes notamment, peuvent également apparaître sous d'autres formes et d'autres occurrences métriques (Charraut, Chouquer, Favory 1994).

A partir de ces avancées, quelques essais ont été tentés sur l'Est lyonnais et ont permis de proposer les hypothèses suivantes (Chouquer 1995/1996) :

Réseau hypothétique "Velin ${ }^{10} \mathrm{~A} ": 23^{\circ}$ Ouest (grille restituée de $710 \mathrm{~m}$ )

Réseau hypothétique "Velin B" : $7^{\circ}$ Ouest (grille restituée de $706 \mathrm{~m}$ )

Réseau hypothétique "Velin C" : 3,3 Est (grille restituée de $710 \mathrm{~m}$ )

L'orientation dite lyonnaise $\left(23,3^{\circ}\right.$ Est) n'est donc plus retenue. En effet, malgré des limites parcellaires actuelles fortes, on n'y retrouve que peu de périodicité. Les décapages livrent en revanche des orientations proches de certaines de ces valeurs. L'orientation nord-sud et ses variantes immédiates se retrouvent dans l'hypothèse de réseau "Velin C" bien que les basculements de quelques degrés vers l'ouest soient plus fréquents que ceux vers l'est. La valeur du "Velin B" est également représentée à plusieurs reprises alors que le "Velin A" ne semble pas trouver d'écho en sous-sol.

A la suite des conclusions de G. Chouquer, plusieurs dizaines d'hectares de terrains ayant servi de supports à l'étude des réseaux hypothétiques du Velin ont été sondés. Ici, le maillage cohérent des sondages explorant non seulement les marges des champs actuels mais aussi les espaces intermédiaires a révélé une limite de la confrontation entre les données de terrain et la carto- et photointerprétation. L'étude des masses parcellaires de Meyzieu "les Taches" et "les Servizières" ou celles de Saint Laurent de Mure "la Grande Cra" avait révélé différents groupes d'occurrences cohérentes dont les écarts variaient de 21 à $37 \mathrm{~m}$. Les évaluations archéologiques situées à 
proximité de ces zones à Meyzieu "les Taches", Meyzieu "les Gaulnes" ou à Saint Laurent de Mure "la Cra" n'ont livré aucune trace fossoyée en sous-sol, les haies vives elles-mêmes n'entamant pas la moraine ou le sol fluvioglaciaire. De ce fait, aucune donnée complémentaire (fossés ou indices de datation) ne peut être versée à ce dossier et étayer ou infirmer les hypothèses émises.

Depuis, la recherche a encore élargi son champ d'étude. Au cours des siècles, de nombreux ensembles parcellaires ont été créés, se sont combinés et ont évolué. Que leur implantation soit orthogonale ou radiale, leurs tracés rigides, souples ou très informels, ces réseaux reflètent une réalité complexe et mouvante que la seule application de grilles strictement normées ne peut révéler (Chouquer dir. 1996 ; Clavel-Lévêque 1993).

La variété et l'imbrication des inclinaisons repérées lors des études archéologiques reflètent bien cette réalité où le fourmillement des informations et une échelle de décapage trop réduite limitent la perception d'éventuels réseaux.

\subsection{L'héritage du cadastre napoléonien}

La superposition des plans de masse et du cadastre napoléonien apporte quelques informations complémentaires. Les décalages ou les dissemblances mis en évidence par cette confrontation sont révélateurs de la sélection des limites d'un terroir sur les vingt derniers siècles mais aussi de la conservation d'un certain nombre d'éléments qui renseignent sur la genèse et parfois l'ancienneté du parcellaire actuel. Les alignements de fosses à galets y trouvent d'ailleurs des éléments d'explication inattendus.

Les traces conservées dans le sous-sol sont beaucoup plus nombreuses que celles lisibles sur le plan napoléonien. Le rapport entre les limites agraires sub-contemporaines en majeure partie composées de sillons interchamps ou de haies et le nombre de fossés issus des décapages " varie de 2,2 pour la zone d'étude 2 à SaintPriest, 2,8 pour les sites de "la Chapelle" et "les Hermières" (zone d'étude 1) à 4 pour les sites de "Sous Trillet" et "l'Epine" (fig. 9, 10 et 11). Cette sélection d'anciens tracés fossoyés s'est effectuée au profit de groupes d'orientations différents aboutissant à la mise en place de masses parcellaires aux tracés originaux et apparemment incohérents.
Ainsi, sur le cadastre napoléonien de "L'Epine", au nord du chemin de Brigneux, une limite de champ trace une ligne brisée donnant naissance à une parcelle de plan très irrégulier. En superposant le plan de masse, il apparaît que ce tracé anguleux correspond à l'imbrication de plusieurs segments de fossés de deux groupes d'orientations différents (Chassieu 1 et Chassieu 2) (fig. 10).

Au sud du site de Chassieu "l'Epine", le chemin "des Charpennes" se termine en impasse. Au décapage, celui-ci se poursuit mais dans le comblement d'un large enclos non daté. Cette desserte rurale refait surface plus au nord sous la forme de radiers de galets déposés sur des tracés fossoyés antiques. Cette section a survécu jusqu'à nos jours mais sous la forme d'une simple limite de champ.

La disparition d'une trace majeure peut néanmoins être complète. Plusieurs décapages ont livré des fossés sous forme de longs tracés parallèles matérialisant le passage d'une ancienne voie ou de chemins creux qui ont intégralement disparu des surfaces actuelles (Saint-Priest "les Feuillis"). De même, à Corbas "BUS", une importante intersection, en discordance avec l'orientation du réseau actuel, a été totalement oblitérée. Deux larges fossés parallèles disparus ont également été découverts à SaintPriest "Illford" associés à un peu de matériel antique.

Le rapport entre les correspondances de tracés lors de la superposition du cadastre avec les plans de masse ${ }^{12}$ est de l'ordre de 3,6 à Saint-Priest et 4 à "l'Epine" et "Trillet". En revanche, il est de 1,75 à "la Chapelle" et "les Hermières" dénotant une stabilité des vestiges linéaires post antiques du groupe Meyzieu 2 (fig. 9, 10 et 11). En élargissant le regard hors de l'emprise décapée, deux autres fossés du même groupe trouvent également un prolongement dans les limites du XIX ${ }^{\mathrm{e}} \mathrm{s}$. (sur "les Hermières"). Sur ces deux derniers sites, la mise en place d'un quadrillage de l'espace dès l'Antiquité a peut-être contribué à fixer durablement certaines de ces limites agraires.

Plus globalement, sur l'ensemble de la zone d'étude 1, il semble que les lieux qui ont fait l'objet d'occupations anciennes (antiques à "l'Epine" ou antiques et médiévales à "la Chapelle") présentent à l'heure actuelle un découpage agraire plus large que les secteurs alentours, où les parcelles sont plus petites, voire laniérées. Sans contredire ce fait, le constat est moins net sur Saint-Priest.

On remarque également l'abandon des fossés des chemins de desserte qui, après maints engorgements suivis de

11 Nombre de limites de parcelles sur le cadastre napoléonien divisé par le nombre de fossés mis au jour sur les décapages, dans la limite de l'emprise archéologique étudiée.

12 Nombre de limites de parcelles sur le cadastre napoléonien divisé par le nombre de fossés mis au jour sur les décapages et correspondant à une limite du XIX ${ }^{c}$ s.. dans la limite de l'emprise archéologique étudiée. 
Fig. 10. Report des fossés sur le cadastre napoléonien à Chassieu "l'Epine", "sous Trillet" et Genas "sous Genas" (zone d'étude I).

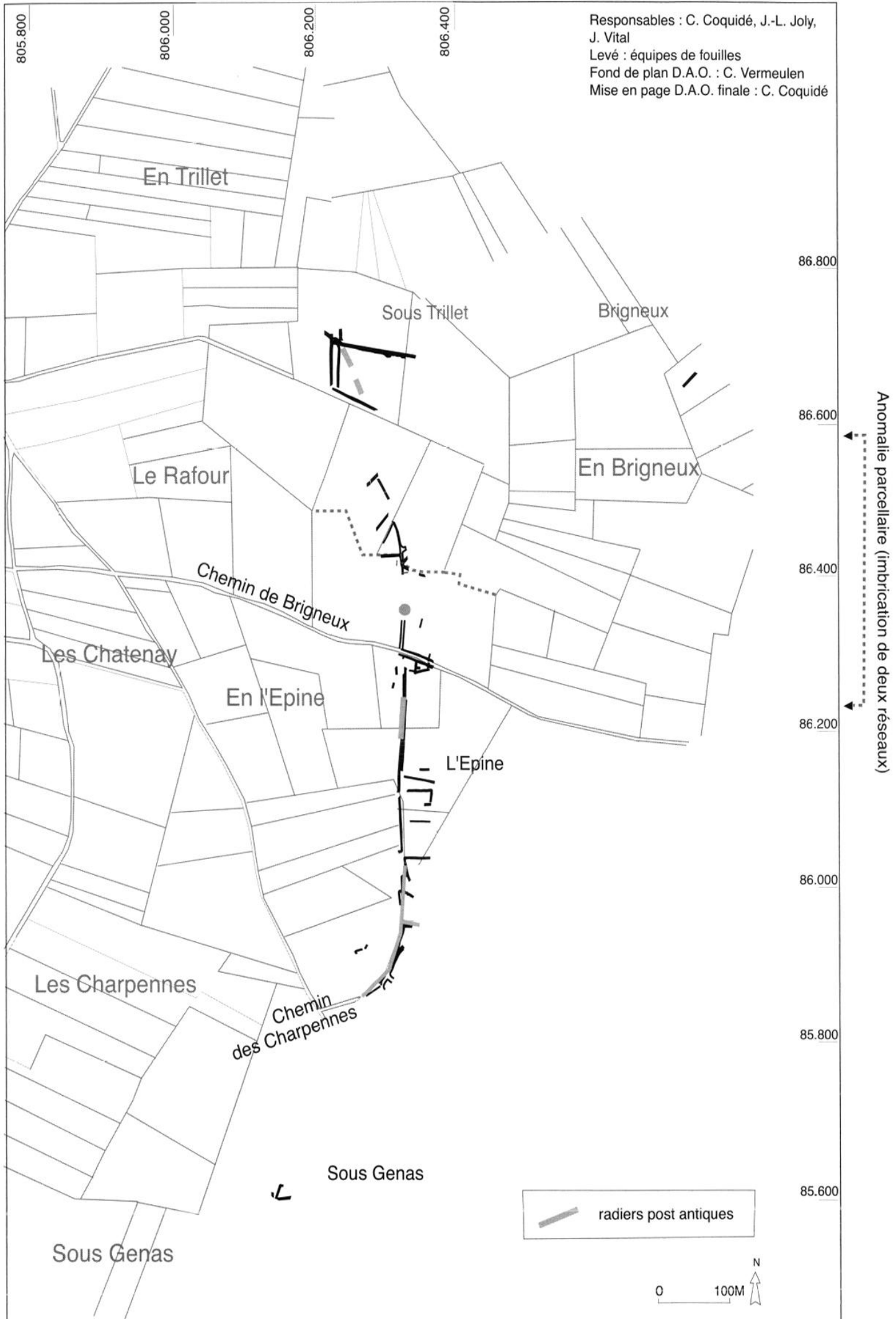

curages, sont finalement remplacés par des haies ou des lignes d'arbres. L'absence d'éléments datant dans les stratigraphies interdit de poser tout jalon chronologique à cette évolution d'une limite fossoyée drainante vers une limite végétale plus superficielle.
Plus que le tracé, c'est son orientation qui survit lors du déplacement de la limite et du redécoupage des surfaces. Ainsi à "L'Epine" et "sous Trillet", les diverses inclinaisons reconnues au travers des groupes d'orientations des fossés se retrouvent dans les limites de champs 


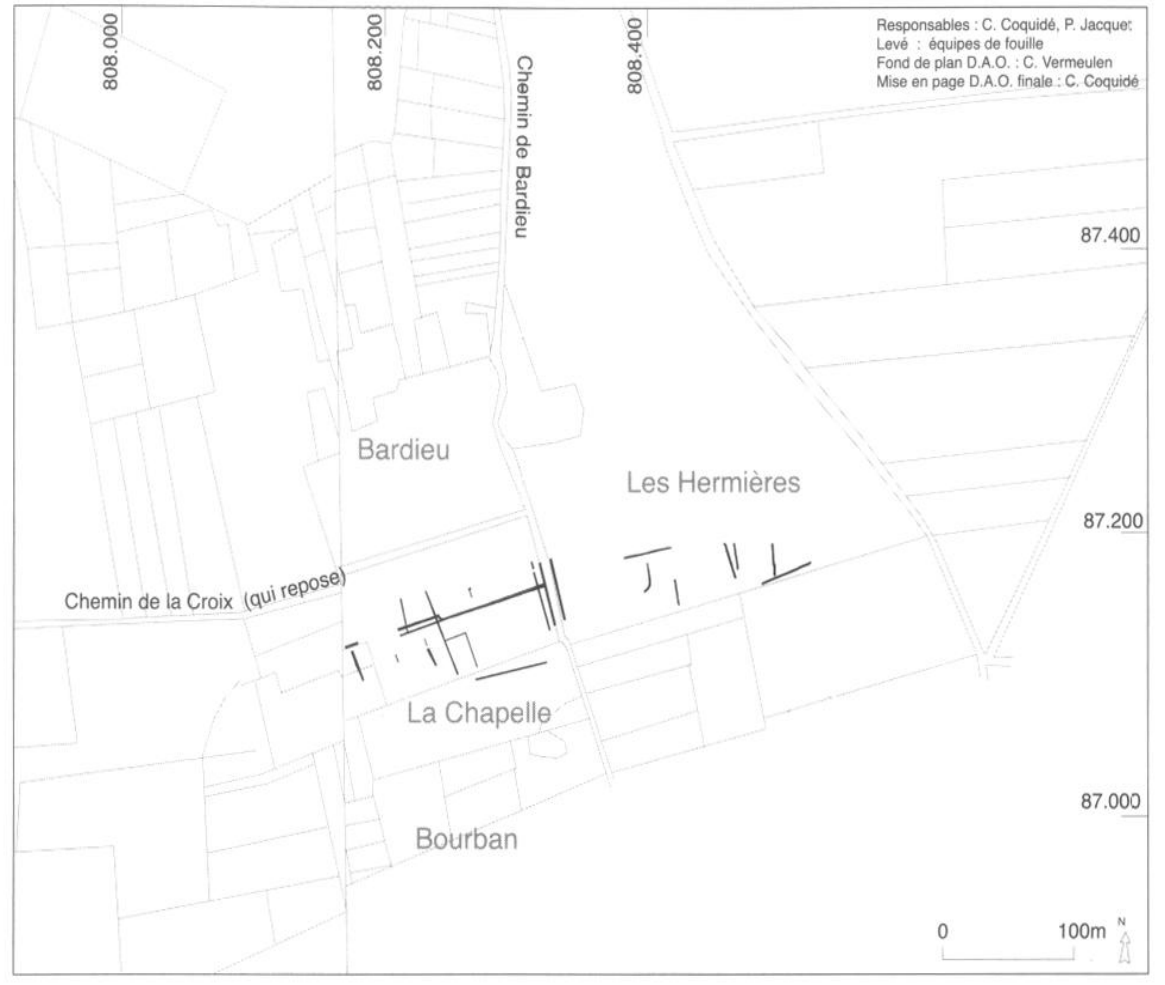

Fig. I I. Report des fossés sur le cadastre napoléonien à Meyzieu "La Chapelle" et "les Hermières" (zone d'étude I).

parus. En revanche, les deux fosses oblongues s'alignent encore selon une inclinaison très proche de celle du cadastre du $\mathrm{XIX}^{\mathrm{e}} \mathrm{s}$.

\section{Conclusion}

L'étude des limites linéaires fossoyées mises au jour lors des décapages archéologiques met en relief l'organisation de l'espace bien au delà des abords immédiats des habitats. Avec le recul des années, il a semblé intéressant de quitter l'échelle du site et d'élargir le regard à la micro-région par le croisement des données issues de deux zones d'études largement décapées, augmentées d'un grand nombre de sites occupant une même entité géomorphologique : la plaine de l'Est lyonnais.

L'amplitude des décapages associée à des indices chronologiques concordants a parfois permis de déceler la présence de schémas d'implantation particuliers composés d'ensembles de fossés plus ou moins organisés, livrant

du paysage actuel, sans que l'une ou l'autre ne prédomine nettement. Une orientation non reconnue en fouille (de 18 à $20^{\circ}$ Ouest) apparaît également (fig. 10). A "La Chapelle" et "Les Hermières", ce sont les fossés orientés à $10^{\circ}$ Ouest (Meyzieu 2) qui se remarquent sur le cadastre napoléonien. L'orientation antique (Meyzieu 1), largement oblitérée par le site médiéval, perd sa prédominance (fig. 11). A Saint-Priest, les deux orientations prépondérantes se disputent encore la prééminence : l'orientation nordsud ("Saint-Priest 1") domine encore, alors que se surimpose l'inclinaison du groupe 2. Les autres orientations se retrouvent d'une manière plus erratique (fig. 9).

Enfin, c'est en ce qui concerne la fonction des fosses à galets que la confrontation du cadastre napoléonien et du plan de masse apporte un élément de réponse inattendu. La ligne de fosses parallèle à un fossé du BUE à Saint-Priest correspond en effet à une limite de champ actuelle (fig. 9). A Saint-Priest "Champ Dolin", le cadastre napoléonien montre quant à lui un fort laniérage des parcelles dont certaines n'atteignent pas dix mètres de large. La superposition du plan de masse avec ce cadastre montre une certaine correspondance entre ce découpage et l'espacement des alignements de fosses (fig. 7). Au lieu-dit "les Taches" en revanche, aucune correspondance avec le cadastre n'est apparue (fig. 8). Si les fosses anguleuses matérialisaient peut-être des retours de parcelles, les limites agraires correspondantes ou même leur orientation ont totalement dis- même parfois une certaine rythmicité (par exemple ceux orientés de $19^{\circ}$ à $23^{\circ}$ ouest). Ces informations n'ayant été recueillies que sur des surfaces relativement réduites à l'échelle de la région, généralement sur un ou deux sites mitoyens, on ne saurait parler de cadastration et encore moins de centuriation. Bornons nous à proposer l'existence de réseaux locaux générés par des habitats, souvent antiques ou parfois médiévaux. Malgré cela, bon nombre de fossés pourtant situés à proximité des occupations humaines ne semblent pas se rattacher à une structuration particulière du paysage et restent (par disparition d'autres traces contemporaines ?) isolés de tout contexte.

Souvent, ce sont les lignes du relief qui sont à l'origine de la grande variété des inclinaisons repérées en fouille. Parmi celles-ci, l'orientation de $10^{\circ}$ Est et surtout celle inclinée vers le nord font figures d'exception puisqu'elles sont mises au jour quelles que soient les lignes de force du paysage. De plus, elles sont toutes deux récurrentes et, simple hasard, préférence culturelle appliquée localement ou plan de plus vaste envergure, leur prégnance sur des zones éloignées surprend.

Avec ou sans planification, l'étude révèle un suivi dans l'aménagement de l'espace, capable d'ćvolucr cn faisant fusionner, au moins localement, contraintes passées et nouvelles. Ainsi, l'imbrication de groupes d'orientations divergentes pose très concrètement la question des modalités de transformation d'un espace donné. Le point d'intersection peut devenir le nœud commun permettant de 
fondre deux réseaux dont le caractère isocline des éléments n'est pas le premier souci. Certains éléments issus de groupes différents peuvent pourtant rester contemporains car répondant à des usages différents (un réseau gérant les espaces agraires associé à un réseau drainant?).

Des exemples tendent également à prouver l'existence de tracés isolés d'inclinaisons variables. Si parmi ceux-ci on note le passage de voies qui semblent indépendantes du cadre local de l'habitat, on remarque également des fossés de large module traversant certaines zones où aucune occupation n'est reconnue. Une fonction de limite de territoire pourrait être évoquée sans que l'échelle de ce dernier ne soit perceptible.

Les hypothèses de cadastre issues de l'interprétation des missions aériennes se perdent dans la variété des orientations reconnues en fouille, à l'exception de l'inclinaison nord-sud qui se distinguc encorc. Lc sous-sol archéologique a en revanche confirmé l'absence de fondement de l'orientation dite lyonnaise $\left(23,3^{\circ} \mathrm{E}\right)$. Autre éclairage à verser au bénéfice des études portant sur l'organisation de l'espace, la confrontation du terrain avec le cadastre napoléonien, héritant de la surimposition de tracés d'orientations différentes au cours des siècles, explique certaines anomalies ou modules contemporains. Enfin, la correspondance de certains alignements de fosses à galets avec des limites de parcelles a permis un nouvel éclairage sur ces vestiges, peut-être à ré-interpréter en terme de divisions agraires.

Il semble bien que, dans les cas présentés, ce soit les plans de masse qui apportent l'information, éclairant la genèse des cartes anciennes, plus que l'inverse. Il apparaît finalement que cette première approche pourrait être prolongée par une confrontation plus fine des données de fouilles avec des documents cartographiques à différentes échelles ${ }^{13}$.

Mais, sur le terrain, c'est avant tout la variété des cas de figure qui se dégage de l'observation des structures linéaires fossoyées, du vestige hors contexte à l'assemblage de différents éléments en un ou plusieurs réseaux plus ou moins cohérents. En tout état de cause, seuls les décapages ne se limitant pas aux marges de l'habitat et intégrant systématiquement à l'étude archéologique les zones investies par les fossés donneront l'opportunité de replacer les sites dans leur espace. Ces ouvertures qui peuvent prendre la forme de tranchées continues et éviter les décapages en aire ouverte coûteux en terme de temps et de stockage de matériaux permettraient de vérifier l'hypothèse d'un habitat générant ses propres limites linéaires, d'en déceler son ou ses réseaux et le cas échéant d'en explorer le rayon.
Des occurrences récurrentes de site à site, et $a$ fortiori indépendantes des contraintes topographiques, pourraient être signalées et les indices datant exposés clairement. Parmi celles-ci, l'orientation au nord mérite une attention particulière. Elle s'associe souvent à l'occupation antique, disparaît même avec elle dans certains sites alors que d'autres plus en accord avec les ruissellements de pente subsistent. La base de l'inclinaison du cadastre B d'Orange, globalement orientée au nord, s'appuie peut-être elle-même sur la vallée du Rhône, axe modelant le paysage (Jung 2000 , p. 352). L'orientation au nord reconnue dans l'Est lyonnais, bien que trop septentrionale pour être associée à cette centuriation, pourrait refléter un choix identique.

Quitter l'échelle du site pour appréhender l'organisation de l'espace au niveau d'une large zone est plus aléatoire. Comment repérer la présence d'éventuels axes structurant le paysage à un moment donné quand les études de terrain restent par nature ponctuelles... Seuls les tracés linéaires abordés par le biais d'un maillage cohérent de sondages pourraient révéler de telles informations à supposer que toutes les limites soient encore perceptibles et livrent des éléments chronologiques fiables.

Le cas des fosses à galets reste à part. Si l'étude a mis en relief un rapport entre certaines de ces structures et une forme de limitation des parcelles observée à distance des habitats, elle n'a pu proposer de contexte plus avancé. Il serait à présent souhaitable, quand cela se présente, de ne pas associer systématiquement ces vestiges à un simple épierrage mais de considérer leur éventuelle implantation par rapport à la fois aux autres éléments de l'organisation de l'espace repérés en fouille et au cadastre napoléonien. L'étude et les analyses palynologiques des comblements d'ensembles cohérents devraient confirmer ou infirmer les hypothèses émises, peut-être même proposer un repère chronologique à leur utilisation.

\section{* INRAP (Lyon-Bron).}

Mes remerciements vont à mes collègues de l'Inrap : Danicl Frascone, Pierre Jacquet, Jean-Luc Joly pour m'avoir autorisée à utiliser les informations contenues dans leurs rapports, Christine Vermeulen pour tout le travail effectué sur les plans des sites de Chassieu-Meyzieu-Décines-Genas, Philippe Hénon et Cécile Ramponi pour m'avoir transmis les données des fouilles des fossés à Saint-Priest "Feuillis" et "BUE", Wojtek Widlak pour les fonds de plans originaux de Saint-Priest, Christine Thollon-Pommerol de la carte archéologique pour son assistance sans faille, sans oublier le soutien des agents du Service Régional de l'Archéologie de Rhône-Alpes chargés de ces dossiers : Anne Le Bot-Helly (conservatrice régionale), Robert Royet (conservateur du patrimoine) et Jacqueline Chastel (ingénieur de recherche). Ce texte doit enfin aux relecteurs de la revue, Frédérique Bertoncello et Jean-Luc Fiches, dont les conseils et les encouragements nous ont été fort utiles.

13 Communication de Gérard Chouquer qui souligne un potentiel inexploité concernant des notions telles que l'héritage et la transmission des formes du paysage. 


\section{BibliographIE}

\section{RAPPORTS ET DOCUMENTS FINAUX DE SYNTHÈSE}

Bedart, Joly 1995 : BEDART (D.), JOLY (J.-L.) - Saint-Priest "Illford". Rapport de sondages, SRA Rhône-Alpes, 1995.

Bouvier et alii 1993 : BOUVIER (A.) et alii - Communay "Charvas". Rapport de fouilles, SRA Rhône-Alpes, 1993.

Bouvier, Verot-Bourrely 2001 : BOUVIER (A.), VEROT-BOURRELY (A.) - A 432, phase diagnosticlévaluations, Villette d'Anthon et Janneyrias (Isère), Pusignan, Colombier-Saugnieu et SaintLaurent-de-Mure (Rhône). Document Final de Synthèse, SRA Rhône-Alpes, 2 vol., 2001.

Chouquer 1995/1996 : CHOUQUER (G.) - Les parcellaires antiques du Velin occidental, tap. Inédit. SRA Rhône-Alpes, 1995/1996.

Constantin, Widlak 1996a : CONSTANTIN (P.), WIDLAK (W.) Corbas "Corbèges". Rapport de sondages, SRA Rhône-Alpes, 1996.

Constantin, Widlak 1996b : CONSTANTIN (P.), WIDLAK (W.) Mions "Pierres blanches". Rapport de sondages, SRA RhôneAlpes, 1996.

Convert, Escoffier 1994 : CONVERT (R.), ESCOFFIER (R.) - Saint Romain de Jalionas "Sambettes". Rapport de sondages, SRA Rhône-Alpes, 1994.

Coquidé 1995a : COQUIDÉ (C.) - Meyzieu "Les Taches". Document Final de Synthèse, SRA Rhône-Alpes, 1995.

Coquidé 1995b : COQUIDÉ (C.) - Saint-Priest "Champ Dolin". Document Final de Synthèse, SRA Rhône-Alpes, 1995.

Coquidé 2002 : COQUIDÉ (C.) - Feyzin "Champ Plantier III". Rapport de sondages, SRA Rhône-Alpes, 2002.

Coquidé, De Sury, Joly 1995 : COQUIDÉ (C.), DE SURY (B.), JOLY (J.-L.) - Saint-Priest "Les Perches". Rapport de sondages, SRA Rhône-Alpes, 1995.

Coquidé, Sandoz 1992 : COQUIDÉ (C.), SANDOZ (G.) - Chassieu "L'Epine". Rapport de fouilles, SRA Rhône-Alpes, 1992.

Coquidé, Vermeulen 1994 : COQUIDÉ (C.), VERMEULEN (C.) Meyzieu "Contournement sud". Rapport de sondages-3e campagne, SRA Rhône-Alpes, 1994.

Coquidé, Vermeulen 1995a : COQUIDÉ (C.), VERMEULEN (C.) Chassieu "Brigneux", Document Final de Synthèse. In : Meyzieu "La Chapelle". SRA Rhône-Alpes, 1995.

Coquidé, Vermeulen 1995b : COQUIDÉ (C.), VERMEULEN (C.) Meyzieu "La Chapelle". Document Final de Synthèse, SRA Rhône-Alpes, 1995.

Dawson, Joly 1992 : DAWSON (C.), JOLY (J.-L.) - Chassieu "Sous Trillet". Rapport de fouilles, SRA Rhône-Alpes, 1992.

Frascone, Staniazsek 1992 : FRASCONE (D.), STANIAZSEK (L.) - Meyzieu "Trillet". Rapport de fouilles, SRA Rhône-Alpes, 1992.

Hénon et alii 1997 : HÉNON (PH.) et alii - Saint-Priest "Les Perches". Document Final de Synthèse, SRA Rhône-Alpes, 1997.

Hénon, Ramponi, Franc (en cours): HÉNON (PH.), RAMPONI (C.), FRANC (O.) - Saint-Priest "ZAC des Feuillis». Document Final de Synthèse, en cours.

Jacquet 1995a : JACQUET (P.) - Meyzieu "Les Hermières". Document Final de Synthèse, SRA Rhône-Alpes, 1995.

Jacquet 1995b : JACQUET (P.) - Meyzieu "Sur Genas". Rapport de fouilles, SRA Rhône-Alpes. 1995.

Joly, De Sury, Franc 1998 : JOLY (J.-L.), DE SURY (B.), FRANC (O.) - Saint-Priest"Boulevard Urbain Est». Rapport de sondages, SRA Rhône-Alpes, 1998.

Joly, Franc, De Sury 1997 : JOLY (J.-L.), FRANC (O.), DE SURY (B.) - Saint-Priest "Zac des Feuillis". Rapport de sondages archéologiques, SRA Rhône-Alpes, 1997.

Joly, Mazuy 2002 : JOLY (J.-L.), MAZUY (D.) - Saint Laurent de Mure "la Cra". Document Final de Synthèse, SRA Rhône-Alpes, 2002.
Joly, Taras 1993 : JOLY (J.-L.), TARAS (M.) - Vénissieux "Vieux Bourg". Document Final de Synthèse, SRA Rhône-Alpes, 1993.

Lurol 1988 : LUROL (J.-M.) - Saint-Priest "Réservoir". Rapport de fouilles, SRA Rhône-Alpes, 1988.

Marchianti 1990 : MARCHIANTI (D.) - Villette d'Anthon "Les Communaux d'Arrézieux". Plan, rapport sommaire, ligne TGV Rhône-Alpes, SRA. Rhône-Alpes, 1990.

Martin, Blaizot 1993 : MARTIN (S.), BLAIZOT (F.) - Marennes "Le Pillon". Rapport de fouilles, SRA Rhône-Alpes, 1993.

Motte 1992 : MOTTE (S.) - Beynost "Grande Croix". Rapport de fouilles, SRA Rhône-Alpes, 1992.

Ramponi et alii 1998 : RAMPONI (C.) et alii - Corbas "B.U.S. Grand Champ". Document Final de Synthèse, SRA Rhône-Alpes, 1998.

Ramponi et alii (en cours) : RAMPONI (C.) et alii - SaintPriest"Boulevard Urbain Est "les Luêpes". Document Final de Synthèse, en cours.

Rebiscoul 2002 : REBISCOUL (A.) - Meyzieu "ZAC des Gaulnes". Rapport de sondages, SRA Rhône-Alpes, 2002.

Royet 1996/1997 : ROYET (R.) - Saint Romain de Jalionas "Le Vernai". Document Final de Synthèse, SRA Rhône-Alpes, 1996/1997.

Vicherd, Baudrand, De Klijn 1981 : VICHERD (G.), BAUDRAND (M.N.), DE KLIJN (H.) - Beligneux "Camp de Chânes". Rapport de fouilles, SRA Rhône-Alpes, 1981.

Vital, Taras, Thieriot 1992 : VITAL (J.), TARAS (M.), THIERIOT (F.) - Genas "Sous Genas". Rapport de fouilles, SRA RhôneAlpes, 1992.

\section{OUVRAGES ET ARTICLES}

Blaizot 2001 : BLAIZOT (F.) - Trois cimetières ruraux de l'Antiquité tardive dans la moyenne vallée du Rhône. Les sites du Pillon à Marennes (Rhône), du Trillet à Meyzieu (Rhône) et des Girardes à Lapalud (Vaucluse). Gallia, 58, 2001, pp. 271-361.

Charraut, Chouquer, Favory 1994 : CHARRAUT (D.), CHOUQUER (G.), FAVORY (F.) - Traitement numérique de l'image. Rythmes et mesures des parcellaires antiques et médiévaux. Archéologia, $\mathrm{n}^{\circ}$ 307, décembre 1994, pp. 24-32.

Chouquer 1996 (sous la direction de) : CHOUQUER (G.) - Les formes du paysage. Tome 1 - Etudes sur les parcellaires. Tome 2 Archéologie des parcellaires. Paris, Ed. Errance, 2 vol., 1996, 224 p. et $263 \mathrm{p}$.

Chouquer, Favory 1980 : CHOUQUER (G.), FAVORY (F.) Contribution à la recherche des cadastres antiques. Paris, 1980, 90 p. (Centre de recherches spécialisées d'histoire ancienne - Equipe de recherche associée au CNRS, vol. 31).

Chouquer, Favory 1991 : CHOUQUER (G.), FAVORY (F.) - Les paysages de l'Antiquité. Terres et cadastres de l'Occident romain. Paris, Ed. Errance, 1991, 243 p.

Clavel-Lévêque 1993 : CLAVEL-LÉVÊQUE (M.) - Territoire, cadastre et environnement. Bilan et perspectives. In : XIIe stage international : De la terre au ciel. Paysages et cadastres antiques. Besançon, 1993, pp. 7-29.

Coquidé, Faure-Boucharlat, Forrest 2001 : COQUIDÉ (C.), FAURE-BOUCHARLAT (E.), FORREST (V.) — « Meyzieu “ La Chapelle" (Rhône) ». In : FAURE-BOUCHARLAT (E.) (dir.) Vivre à la campagne au Moyen Âge. L'habitat rural du Ve au XIIle s. (Bresse, Lyonnais, Dauphiné) d'après les données archéologiques. Lyon, SRA Rhône-Alpes, 2001, pp. 282-291 (DARA n ${ }^{\circ}$ 21).

Coquidé, Vermeulen 1999 : COQUIDÉ (C.), VERMEULEN (C.) Chassieu-Genas " L'Epine" (Rhône). "Evolution d'un habitat rural du Ir s. av. J.-C. au III" s. ap. J.-C.". RANarb. 32. 1999. pp. 197-244. 
Hénon et collab. 1999 : HÉNON (Ph.) et collab. - Structures domestiques et espaces villageois du Ve millénaire au VIIle siècle avant notre ère. L'apport des fouilles de la «ZAC des Perches » (Rhône). Bilan documentaire. In : Premières journées d'Histoire en Velin. Cercle Julius Victor. Saint-Priest, 20 et 21 novembre 1999, pp. 2965.
Jung 2000 : JUNG (C.) - Pérennité et mutations dans l'organisation de paysages agraires : le Tricastin et le terroir de Lattes. RANarb, 33, 2000, pp. 351-380.

Monnier 1990 : MONNIER (J.) - La Dent. Site gallo-romain à Meyzieu (Rhône). Découvertes archéologiques à Genas, Azieu et Décines-Charpieu. Lyon, 1990, 186 p. (CERGR, nlle. série n 8). 